\title{
Management and outcomes in critically ill nonagenarian versus octogenarian patients
}

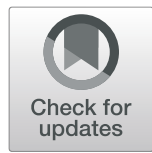

Raphael Romano Bruno ${ }^{1 \dagger}$, Bernhard Wernly ${ }^{2,3+}$, Malte Kelm ${ }^{1,4}$, Ariane Boumendil ${ }^{5}$, Alessandro Morandi ${ }^{6,7}$, Finn H. Andersen ${ }^{8,9}$, Antonio Artigas ${ }^{10}$, Stefano Finazzi ${ }^{11}$, Maurizio Cecconi ${ }^{12}$, Steffen Christensen ${ }^{13}$,

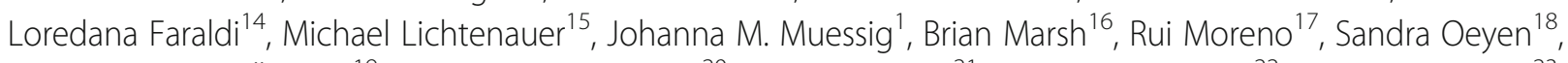
Christina Agvald Öhman ${ }^{19}$, Bernardo Bollen Pinto ${ }^{20}$, Ivo W. Soliman ${ }^{21}$, Wojciech Szczeklik ${ }^{22}$, Andreas Valentin ${ }^{23}$, Ximena Watson ${ }^{24}$, Susannah Leaver ${ }^{25}$, Carole Boulanger ${ }^{26}$, Sten Walther ${ }^{27}$, Joerg C. Schefold ${ }^{28}$, Michael Joannidis ${ }^{29}$, Yuriy Nalapko ${ }^{30}$, Muhammed Elhadi ${ }^{31}$, Jesper Fjølner ${ }^{32}$, Tilemachos Zafeiridis ${ }^{33}$, Dylan W. De Lange ${ }^{21}$, Bertrand Guidet ${ }^{5,34,35}$, Hans Flaatten ${ }^{36,37}$, Christian Jung ${ }^{1 *}$ and on behalf of the VIP2 study group

\begin{abstract}
Background: Intensive care unit (ICU) patients age 90 years or older represent a growing subgroup and place a huge financial burden on health care resources despite the benefit being unclear. This leads to ethical problems. The present investigation assessed the differences in outcome between nonagenarian and octogenarian ICU patients.

Methods: We included 7900 acutely admitted older critically ill patients from two large, multinational studies. The primary outcome was 30-day-mortality, and the secondary outcome was ICU-mortality. Baseline characteristics consisted of frailty assessed by the Clinical Frailty Scale (CFS), ICU-management, and outcomes were compared between octogenarian (80-89.9 years) and nonagenarian ( $\geq 90$ years) patients. We used multilevel logistic regression to evaluate differences between octogenarians and nonagenarians.

Results: The nonagenarians were $10 \%$ of the entire cohort. They experienced a higher percentage of frailty (58\% vs $42 \% ; p<0.001)$, but lower SOFA scores at admission ( $6 \pm 5$ vs. $7 \pm 6 ; p<0.001)$. ICU-management strategies were different. Octogenarians required higher rates of organ support and nonagenarians received higher rates of lifesustaining treatment limitations ( $40 \%$ vs. $33 \% ; p<0.001)$. ICU mortality was comparable ( $27 \%$ vs. $27 \% ; p=0.973$ ) but a higher 30 -day-mortality ( $45 \%$ vs. $40 \% ; p=0.029$ ) was seen in the nonagenarians. After multivariable adjustment nonagenarians had no significantly increased risk for 30-day-mortality (aOR 1.25 (95\% Cl 0.90-1.74; $p=0.19)$ ).

Conclusion: After adjustment for confounders, nonagenarians demonstrated no higher 30-day mortality than octogenarian patients. In this study, being age 90 years or more is no particular risk factor for an adverse outcome. This should be considered- together with illness severity and pre-existing functional capacity - to effectively guide triage decisions.
\end{abstract}

Trial registration: NCT03134807 and NCT03370692.

Keywords: Octogenarians, Nonagenarians, Frailty, Intensive care medicine, Outcome

\footnotetext{
* Correspondence: Christian.Jung@med.uni-duesseldorf.de

${ }^{\dagger}$ Raphael Romano Bruno and Bernhard Wernly contributed equally to this work.

'Department of Cardiology, Pulmonary Diseases, and Vascular Medicine, Medical Faculty, Heinrich Heine University of Duesseldorf, Moorenstraße 5, 40225 Duesseldorf, Germany

Full list of author information is available at the end of the article
}

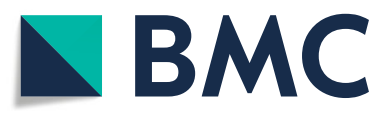

(c) The Author(s). 2021 Open Access This article is licensed under a Creative Commons Attribution 4.0 International License, which permits use, sharing, adaptation, distribution and reproduction in any medium or format, as long as you give appropriate credit to the original author(s) and the source, provide a link to the Creative Commons licence, and indicate if changes were made. The images or other third party material in this article are included in the article's Creative Commons licence, unless indicated otherwise in a credit line to the material. If material is not included in the article's Creative Commons licence and your intended use is not permitted by statutory regulation or exceeds the permitted use, you will need to obtain permission directly from the copyright holder. To view a copy of this licence, visit http://creativecommons.org/licenses/by/4.0/. The Creative Commons Public Domain Dedication waiver (http://creativecommons.org/publicdomain/zero/1.0/) applies to the data made available in this article, unless otherwise stated in a credit line to the data. 


\section{Introduction}

The proportion of older patients has increased significantly over time. In 2030, there will be more than 30 million people over the age of 90 (nonagenarians) in 35 industrialised countries [1]. Consequently, health care providers nowadays perform medical procedures on very old patients (from surgery to oncological therapies), which were previously considered unfeasible because of age or age-related deterioration in physical and mental performance [2]. Similarly, the rate of older patients $(\geq 80$ years) in intensive care units (ICU) is increasing [3-6]. Today, older patients utilise a disproportionate amount of health care resources compared to their relative proportion of the total population [3, 7].

In particular, the extent to which "old age" per se is a risk factor and the extent to which different groups of old patients differ from one another regarding the prognosis is the subject of continuing debate. Older patients suffer worse outcomes than younger patients undergoing intensive care $[8,9]$, but some studies failed to establish age as an independent predictor of mortality in older ICU patients $[10,11]$. However, most prognostic studies demonstrated an almost linear relationship between chronological age and mortality after the age of 40 [12]. In this respect, patients ageing 80 years and more represent a particular challenge to intensive care medicine $[13,14]$. Still, there are no large studies that further differentiate this group of very old ICU patients and it is unclear if being a nonagenarian is a risk factor for adverse outcomes. We hypothesize that critically ill nonagenarians have an elevated 30-day mortality compared to octogenarians. To address this hypothesis, we performed a retrospective cohort study comprised of two large, multinational prospective observational cohorts [13-15]. This post-hoc analysis combined data from the VIP-1 and VIP-2 studies to compare octoand nonagenarians regarding 30-day mortality (primary outcome) and ICU mortality (secondary outcome), the distribution of risk factors, and the intensive care management [13-15].

\section{Methods}

\section{Study subjects}

The very old intensive care patients (VIP) studies, VIP1 and VIP2, were prospective, multi-centre studies, registered on ClinicalTrials.gov (ID: NTC03134807, NCT03370692). Both studies included very old intensive care patients (VIPs), defined as patients admitted to an ICU and aged 80 years or older. The main results from these studies have been published previously [13, 14, 16, 17]. In summary, for both studies, each participating ICU could include either consecutive patients admitted over a six-month period or the first 20 consecutive patients fulfilling the inclusion criteria (all patients aged 80 years or older). The data collection for VIP1 took place between October 2016 and
February 2017 and between May 2018 to May 2019 for VIP2. Both studies used similar inclusion criteria as described elsewhere [13]. Informed consent was obtained from study participants. Local ethical committees might have waived the need of informed consent.

In this post-hoc analysis of these two studies, all patients admitted acutely (non-electively) with complete data on age, gender, clinical frailty score (CFS), sequential organ failure assessment (SOFA) score, and ICU mortality were included. For this study, the elective patients included in VIP1 were excluded as their outcomes differ significantly compared with those admitted acutely, as previously shown [18]. The primary endpoint of this study was ICU-mortality, and the secondary endpoint was 30-day-mortality.

Scales, scores, and limitations in life-sustaining therapy The SOFA score was recorded on admission; it could be calculated manually or using an online calculator. Frailty was assessed by the clinical frailty scale (CFS). The CSF distinguishes nine classes of frailty from very fit (CFS 1) to terminally ill (CFS 9). The respective visual and simple description for this assessment tool was used with permission [19-21].

The Katz Activities of Daily Living (Katz ADL) scale is a widely used graded instrument to assess disability in chronically ill or older patients. It evaluates six primary and psychosocial functions: bathing, dressing, going to the toilet, transferring, feeding, and continence. The patient receives 1 point for every independent and 0 for every dependent activity $(6=$ independent patient, $0=$ very dependent patient). For the patients in the VIP2 trial, disability was defined by Katz ADL score $\leq 4$.

For cognitive decline, VIP2 utilised the Short form of Informant Questionnaire on Cognitive Decline in the Elderly (IQCODE). IQCODE is a questionnaire, completed by carers, with 16 questions about cognitive decline over the past 10 years. For each question, 1 to 5 points can be assigned. An average of 3 points per question is considered "normal". A cumulative IQCODE of $\geq 3.5$ is regarded as "cognitive decline" [19-21].

The burden of co-morbidity was assessed using the co-morbidity and polypharmacy score (CPS) [22]. The CPS calculates the total number of chronic diagnoses and drugs taken. Standard ICU procedures were also documented.

In addition, limitations of therapy, such as withholding or withdrawing treatment, were recorded. Withholding life-sustaining therapy (e.g. mechanical ventilation, renal replacement therapy, cardiopulmonary resuscitation) was defined as not performing a measure that was indicated; withdrawing was defined as stopping any kind of life-sustaining therapy. All these decisions were at the discretion of the treating physicians and documented 
according to international recommendations. VIP2 recorded the exact date of treatment limitation, but VIP1 did not give specific details. Thus, the present analysis used withholding or withdrawing treatment as binary information at any time during the ICU-stay.

\section{Statistical analysis}

Post-hoc power calculations using the 7110 octogenarians and 790 nonagenarians, primary outcome event rates of $40 \%$ versus $45 \%$, and an alpha of 0.05 , the power of the study to detect differences in 30-day mortality is $77 \%$. Continuous data points are expressed as median \pm interquartile range. Differences between independent groups were calculated using the Mann Whitney U-test. Categorical data are expressed as numbers (percentage). The chi-square test was applied to calculate differences between groups. Sensitivity analysis, analysing only patients with SOFA scores below the 75th percentile SOFA score of 10 (i.e. all patients with SOFA < 10) was performed. Univariable and multivariable logistic regression analysis was performed to assess associations with treatment limitations and mortality. Odds ratios (OR) and adjusted odds ratios (aOR) with respective 95\% confidence intervals $(\mathrm{CI})$ were calculated. Two sequential random effects, multilevel logistic regression models were used to evaluate the impact of being a nonagenarian on ICU- and 30-days- mortality. All patients with valid data on ICU-mortality were included. First, a baseline model with being nonagenarian as a fixed effect and ICU as random effect (model-1) was fitted. Second, to model-1, patient characteristics (SOFA, CFS, sex) (model-2) were added to the model. Adjusted odds ratios $(\mathrm{aOR})$ with respective 95\% confidence intervals (CI) were calculated. Sensitivity analysis, analysing only patients with and without any treatment limitation was performed. All tests were two-sided, and a $p$-value of $<0.05$ was considered statistically significant. SPSS version 23.0 (IBM, USA) and MedCalc Statistical Software version 19.1.3 (MedCalc Software bv, Ostend, Belgium; https://www. medcalc.org; 2019) were used for all statistical analyses.

\section{Results}

\section{Study population}

This study included 7900 patients. $10 \%$ of the patients were nonagenarians. Table 1 displays the baseline characteristics of nonagenarians versus octogenarians. Nonagenarians were predominantly female (57\% versus $46 \%, p<0.001$ ), evidenced higher rates of frailty ( $58 \%$ vs $42 \% ; p<0.001)$, disability ( $44 \%$ vs. $26 \% ; p<0.001)$ and cognitive decline $(50 \%$ vs. $31 \% ; p<0.001)$ but lower SOFA scores at admission $(6 \pm 5$ vs. $7 \pm 6 ; p<0.001)$. Specific ICU-treatment strategies were used, with octogenarians receiving higher rates of organ support (renal replacement therapy, mechanical ventilation, vasoactive drugs), while for nonagenarians there were higher rates of treatment limitation ( $40 \%$ vs. $33 \%$; $p<0.001$; Table 1$)$. After discharge from the ICU, most patients had a treatment limitation; 1053 octogenarians $(55 \%$ of all octogenarians leaving the ICU alive) and 182 (85\%) nonagenarians left the ICU with treatment limitations in place.

\section{Survival analysis in the total cohort}

The overall ICU mortality was $27 \%(N=2134$ of 7900 patients), the 30-day-mortality was $39 \%(N=3080$ of 7555 patients). Compared to the octogenarians the nonagenarians had a similar ICU mortality $(27 \%$ vs. 27\%; $p=0.973)$, but a higher 30-day-mortality (45\% vs. $40 \% ; p=0.029$, Fig. 1$)$. Nonagenarians showed a significantly longer length of ICU-stay $(84 \mathrm{~h}$ versus 54 h, $p<0.001$ ).

\section{Comparison of nonagenarians versus octogenarians in the multilevel logistic regression models}

After the adjustment for the ICU cluster as a random effect (model-1), nonagenarians had an increased risk for withholding life-sustaining therapy (aOR 1.54 (95\% CI 1.22-1.94; $\mathrm{p}=<0.001)$ ), but not for withdrawal (aOR 1.03 (95\% CI 0.77-1.39; p=0,82)). Nonagenarians received significantly less mechanical ventilation, renal replacement therapy and vasoactive drugs. There was no difference between both age groups regarding the use of mechanical ventilation, vasopressors, and ICU-mortality, but an increased risk for 30-day-mortality (aOR 1.39 (95\% CI 1.13-1.72); $p=0.002$ ). After adding patientspecific confounders (model-2), nonagenarians demonstrated no significant risks compared to octogenarians (Table 2)

\section{Discussion}

This study examines the largest multi-centre prospectively recruited group of intensive care patients of 90 years and older published to date. Nonagenarians differ in their baseline risk distribution, management, and clinical outcomes from octogenarians. Nonagenarians had higher rates of frailty, cognitive impairment, and disability. However, when compared with octogenarians, nonagenarians had a lower illness severity and required less organ support. After adjustment for relevant confounders, the 30-day mortality did not differ between both groups.

Our results are in line with other studies looking at older ICU patients: Fuchs et al. evaluated a cohort of more than 7000 surgical and medical ICU patients and found age, especially above 75 years, to be an independent risk factor for mortality $[9,23]$. In a large retrospective analysis of $1,807,531$ patients admitted to an ICU between 1997 and 2016, Jones et al. reported increased 
Table 1 Baseline characteristics in the total cohort, nonagenarians versus octogenarians

\begin{tabular}{|c|c|c|c|}
\hline & \multirow{2}{*}{$\begin{array}{l}\text { nonagenarians } \\
n=790\end{array}$} & \multirow{2}{*}{$\begin{array}{l}\text { octogenarians } \\
n=7110\end{array}$} & \multirow[t]{2}{*}{$p$-value } \\
\hline & & & \\
\hline Male sex n (\%) & $339(43 \%)$ & $3812(54 \%)$ & $<0.001$ \\
\hline \multicolumn{4}{|l|}{ Age } \\
\hline median $( \pm \mid \mathrm{QR})$ & $91(90-93)$ & $83(81-86)$ & $<0.001$ \\
\hline \multicolumn{4}{|l|}{ Frailty Score - CFS } \\
\hline median $( \pm \mid \mathrm{QR})$ & $5(4-6)$ & $4(3-6)$ & $<0.001$ \\
\hline Frailty (CFS > 4) n (\%) & $454(58)$ & $2962(42)$ & $<0.001$ \\
\hline \multicolumn{4}{|l|}{ ADL } \\
\hline median $( \pm \mid \mathrm{QR})$ & $5(3-6)$ & $6(4-6)$ & $<0.001$ \\
\hline Disability (ADL $\leq 4)$ & $151(44)$ & $805(26)$ & $<0.001$ \\
\hline \multicolumn{4}{|l|}{ IQCODE } \\
\hline median $( \pm \mid \mathrm{QR})$ & $3.5(3-4)$ & $3.2(3-4)$ & $<0.001$ \\
\hline Cognitive Decline (IQCODE $\geq 3.5$ ) & $149(50)$ & $812(31)$ & $<0.001$ \\
\hline SOFA score & $6(4-9)$ & $7(4-10)$ & $<0.001$ \\
\hline \multicolumn{4}{|l|}{ median $( \pm \mid \mathrm{QR})$} \\
\hline \multicolumn{4}{|l|}{ ICU length of stay (hours) } \\
\hline median $( \pm \mid \mathrm{QR})$ & $84(24-117)$ & $54(37-186)$ & $<0.001$ \\
\hline Treatment withdrawn and/or withheld (\%) & $312(40)$ & $2302(33)$ & $<0.001$ \\
\hline NIV n (\%) & $168(21)$ & $1794(23)$ & 0.03 \\
\hline Intubation n (\%) & $324(41)$ & $3685(52)$ & $<0.001$ \\
\hline Renal replacement therapy n (\%) & $33(4)$ & $816(12)$ & $<0.001$ \\
\hline Vasoactive drugs n (\%) & $414(52)$ & $4179(59)$ & 0.002 \\
\hline Admission diagnosis n (\%) & & & $<0.001$ \\
\hline Respiratory failure & $155(20)$ & $1745(23)$ & \\
\hline Circulatory failure & $136(17)$ & $968(14)$ & \\
\hline Combined circulatory \& respiratory failure & $104(13)$ & $825(12)$ & \\
\hline Sepsis & $74(9)$ & $966(14)$ & \\
\hline Multitrauma w/o Head Injury & $23(3)$ & $128(2)$ & \\
\hline Trauma with Head Injury & $18(2)$ & $124(2)$ & \\
\hline Head Injury & $29(4)$ & $166(2)$ & \\
\hline Intoxication & $1(<1)$ & $36(<1)$ & \\
\hline Cerebral Injury (Non-Traumatic) & $38(5)$ & $469(7)$ & \\
\hline Emergency Surgery & $91(12)$ & $817(12)$ & \\
\hline Other & $91(12)$ & $866(12)$ & \\
\hline
\end{tabular}

CFS Clinical Frailty Scale, SOFA Sequential Organ Failure Assessment, ADL Activity of Daily Life measured with the Katz index, IQCODE Informant Questionnaire on COgnitive Decline in the Elderly, ICU Intensive Care Unit, NIV Non-Invasive Ventilation, SD Standard Deviation

mortality in patients older than 84 years, although they had a similar illness severity at ICU admission compared to younger patients [23]. Conversely, in a study evaluating 5882 patients after cardiac arrest, age alone was only a weak predictor of mortality [24]. In a recent study by Roedl et al., a survival rate of $46 \%$ with a good neurological outcome was reported for nonagenarians after cardiac arrest [11]. Recently, Druwé et al. performed a subgroup analysis on out-of-hospital cardiac arrests with a special interest in the resuscitation attempts in octogenarians: Most physicians considered cardiopulmonary resuscitation to be appropriate even in older patients with poor outcome perspectives [25]. Furthermore, in another study by Becker et al., the ICU mortality of nonagenarians was low at 30\% and, importantly, the oneyear survival was $50 \%$, indicating outcomes "better than expected" in nonagenarians [26]. Of note, the study by Becker et al. was a single-centre study, and the number 
A

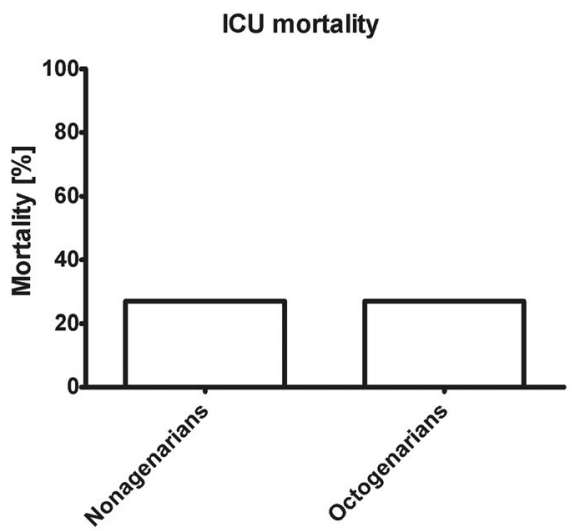

B

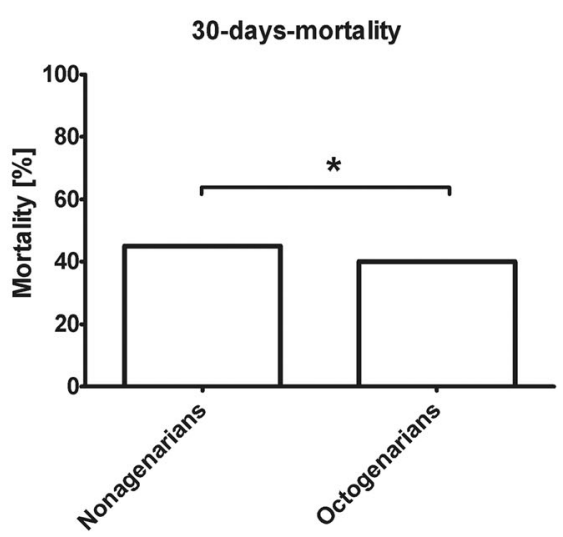

Fig. 1 Comparison of 30-day and ICU-mortality. A: ICU-mortality [\%], B: 30 -day-mortality [\%]. ${ }^{*}=p<0.05$

of patients who received vasoactive drugs was lower when compared to the patients in our multi-centre study. Therefore, we propose the higher mortality rates reported in the present study may be more representative of a "real-world scenario".

Demoule et al. performed a matched case-control study in 36 nonagenarians admitted to an ICU. They were matched according to sex with 72 controls: ICU admissions chosen from the 20- to 69-year age range. They found no differences in the reason for admission, but nonagenarians suffered significantly less from pre-existing co-morbidities. Advanced life- support interventions were used equally. ICU and intra-hospital mortality, as well as the length of stay, did not differ significantly between nonagenarians and the control group [27]. Despite differences in the absolute length of stay, the trend of a shorter length of stay for older (nonagenarian) intensive care patients is consistent with previous studies [28].

Interestingly, being a nonagenarian was independently associated with the decision for withholding lifesustaining therapy, but not for withdrawing it. After adjustment for patient characteristics, nonagenarians evidenced no particular risk for treatment limitations

Table 2 Associations of primary exposure (being nonagenarian) with mortality and management strategies in a multilevel logistic regression model

\begin{tabular}{|c|c|c|c|c|c|}
\hline & octogenarians & nonagenarians & $p$-value & model-1 & model-2 \\
\hline Treatment withheld & $27 \%(1945)$ & $35 \%(279)$ & $<0.001$ & $\begin{array}{l}\text { aOR } 1.54 \\
(95 \% \text { Cl } 1.22-1.94 ; p=<0.001)\end{array}$ & $\begin{array}{l}\text { aOR } 0.95 \\
(95 \% \text { Cl } 0.67-1.36 ; p=0.79)\end{array}$ \\
\hline Treatment withdrawn & 14\% (1026) & $13 \%(102)$ & 0.24 & $\begin{array}{l}\text { aOR } 1.03 \\
(95 \% \mathrm{Cl} 0.77-1.39 ; p=0.82)\end{array}$ & $\begin{array}{l}\text { aOR } 0.73 \\
(95 \% \text { Cl } 0.48-1.10 ; p=0.13)\end{array}$ \\
\hline NIV & $25 \%(1794)$ & $21 \%(168)$ & 0.014 & $\begin{array}{l}\text { aOR } 0.79 \\
(95 \% \text { Cl } 0.61-1.03 ; p=0.08)\end{array}$ & $\begin{array}{l}\text { aOR } 0.85 \\
(95 \% \text { Cl } 0.59-1.22 ; p=0.36)\end{array}$ \\
\hline Mechanical Ventilation & $52 \%(3685)$ & $41 \%(324)$ & $<0.001$ & $\begin{array}{l}\text { aOR } 0.72 \\
(95 \% \text { Cl } 0.56-0.93 ; p=0.01)\end{array}$ & $\begin{array}{l}\text { aOR } 1.26 \\
(95 \% \text { Cl } 0.85-1.87 ; p=0.26)\end{array}$ \\
\hline RRT & $11 \%(816)$ & $4 \%(33)$ & $<0.001$ & $\begin{array}{l}\text { aOR } 0.32 \\
(95 \% \text { Cl } 0.19-0.53 ; p=<0.001)\end{array}$ & $\begin{array}{l}\text { aOR } 0.55 \\
(95 \% \text { Cl } 0.28-1.08 ; p=0.08)\end{array}$ \\
\hline Vasoactive drugs & $59 \%(4179)$ & $52 \%(414)$ & $<0.001$ & $\begin{array}{l}\text { aOR } 0.74 \\
(95 \% \mathrm{Cl} 0.58-0.95 ; p=0.017)\end{array}$ & $\begin{array}{l}\text { aOR } 0.90 \\
(95 \% \mathrm{Cl} 0.60-1.35 ; p=0.62)\end{array}$ \\
\hline 30d Mortality & $40 \%(2743)$ & $44 \%(337)$ & 0.029 & $\begin{array}{l}\text { aOR } 1.39 \\
(95 \% \text { Cl } 1.13-1.72) ; p=0.002)\end{array}$ & $\begin{array}{l}\mathrm{aOR} 1.25 \\
(95 \% \mathrm{Cl} 0.90-1.74 ; p=0.19)\end{array}$ \\
\hline ICU-Mortality & 27\% (1921) & $27 \%(213)$ & 0.97 & $\begin{array}{l}\text { aOR } 1.10 \\
(95 \% \text { Cl } 0.87-1.40) ; p=0.43)\end{array}$ & $\begin{array}{l}\text { aOR } 0.91 \\
(95 \% \mathrm{Cl} 0.63-1.32 ; p=0.63)\end{array}$ \\
\hline
\end{tabular}

NIV Non-Invasive Ventilation, RRT renal replacement therapy, ICU Intensive Care Unit, aOR Adjusted Odds Ratio, 95\% CI 95\% Confidence Interval Model 1 - ICU cluster (the patient's individual ICU) as random effect

Model 2 - Model 1 plus patient level (SOFA, CFS, age, sex) 
compared to octogenarians. These findings contradict the usual expectation that physicians in general tend to be more reluctant to provide organ support to nonagenarians compared to similarly sick octogenarians. In nonagenarians, ICU re-triage should be emphasised: after an initial intensive care treatment for up to $48 \mathrm{~h}$, patients should be critically evaluated in cooperation with their family and/ or carers and discharged to a normal ward for best-supportive care if further intensive care seems unethical, unjustified, or unlikely to improve outcomes. However, modern intensive care medicine is not limited to life-sustaining measures. Even beyond invasive ventilation, renal replacement therapy or cardiopulmonary resuscitation, intensive care medicine can provide valuable treatment for the patient, which might be intensified palliative therapy. Based on our data, being a nonagenarian does not represent a particular risk factor for adverse outcomes. Application of ICU re-triage could help to reduce the economic burden of ICU care in very old patients, in addition to unethical intensive care and distress caused to health care providers.

Mortality was similar between octogenarians and nonagenarians at ICU discharge and after 30 days. The long-term outcomes of the VIP2 study are awaited and will answer the question of whether this effect remains stable further over time.

An important limitation is, that we have no information about pre-ICU triage decisions, although this might be an important factor for the differences in disease illness scores and frailty between nonagenarians and octogenarians. Furthermore, this study only provides detailed information up to ICU-discharge and there was a significant rise in mortality during the 30 days after ICU-discharge, but we do not have detailed data on decisions made and developments during this period. Another limitation is that no a priori sample size calculation was made to detect a difference in the mortality between nonagenarians and octogenarians. Our post-hoc power calculation shows that the present study is likely underpowered for the primary outcome, and thus the reporting results that are at a higher risk of false positive results. However, this was counterbalanced by using a multilevel model to adjust for relevant confounders.

\section{Conclusion}

Nonagenarian ICU patients demonstrated higher rates of frailty but had less acute organ dysfunction than octogenarians. After adjustment for multiple relevant confounders, nonagenarians did not suffer from worse outcomes compared to octogenarian ICU patients. Rather than being a nonagenarian, the severity of illness, functional capacity - and of course the patients' will should guide triage decisions.
Acknowledgements VIP-2-STUDY GROUP:

\begin{tabular}{llll}
\hline Hospital & City & ICU & Name \\
& & \\
\hline
\end{tabular}

Austria

\begin{tabular}{lcll}
\hline $\begin{array}{l}\text { Medical } \\
\text { University } \\
\text { Innsbruck }\end{array}$ & Innsbruck & $\begin{array}{l}\text { Division of Intensive Care } \\
\text { and Emergency Medicine, } \\
\text { Department of Internal } \\
\text { Medicine }\end{array}$ & $\begin{array}{l}\text { Michael } \\
\text { Joannidis }\end{array}$ \\
$\begin{array}{l}\text { Medical } \\
\text { University Graz }\end{array}$ & Graz & $\begin{array}{l}\text { Allgemeine Medizin } \\
\text { Intensivstation }\end{array}$ & Philipp Eller \\
$\begin{array}{l}\text { Medical } \\
\text { University of } \\
\text { Innsbruck }\end{array}$ & Innsbruck & $\begin{array}{l}\text { Department of Neurology, } \\
\text { Neurocritical Care Unit }\end{array}$ & $\begin{array}{l}\text { Raimund } \\
\text { Helbok }\end{array}$ \\
$\begin{array}{l}\text { Hospital of St. } \\
\text { John of God }\end{array}$ & Wienna & ICU B5 & René \\
& & & Schmutz
\end{tabular}

Belgium

\begin{tabular}{|c|c|c|c|}
\hline $\begin{array}{l}\text { AZ Maria } \\
\text { Middelares Ghent }\end{array}$ & Ghent & $\begin{array}{l}\text { Department of } \\
\text { Intensive Care }\end{array}$ & Joke Nollet \\
\hline $\begin{array}{l}\text { OLVrouw Hospital } \\
\text { Aalst }\end{array}$ & Aalst & $\begin{array}{l}\text { Department of } \\
\text { Intensive Care }\end{array}$ & $\begin{array}{l}\text { Nikolaas de } \\
\text { Neve }\end{array}$ \\
\hline AZ Sint-Lucas & Ghent & $\begin{array}{l}\text { Department of } \\
\text { Intensive Care }\end{array}$ & $\begin{array}{l}\text { Pieter De } \\
\text { Buysscher }\end{array}$ \\
\hline $\begin{array}{l}\text { Ghent University } \\
\text { Hospital }\end{array}$ & Ghent & $\begin{array}{l}\text { Department of } \\
\text { Intensive Care }\end{array}$ & Sandra Oeyen \\
\hline AZ Sint-Blasius & Dendermonde & $\begin{array}{l}\text { Department of } \\
\text { Intensive Care }\end{array}$ & $\begin{array}{l}\text { Walter } \\
\text { Swinnen }\end{array}$ \\
\hline
\end{tabular}

Croatia

\begin{tabular}{|c|c|c|}
\hline $\begin{array}{l}\text { Clinical Hospital } \\
\text { Centre Split }\end{array}$ & Split & $\begin{array}{l}\text { Institute for Intensive } \\
\text { Medicine }\end{array}$ \\
\hline
\end{tabular}

Denmark

\begin{tabular}{llll}
\hline Bispebjerg Hospital & Copenhagen & Intensiv Terapi Afsnit & $\begin{array}{l}\text { Anders } \\
\text { Bastiansen }\end{array}$ \\
$\begin{array}{l}\text { Regionshospitalet } \\
\text { Randers }\end{array}$ & Randers & ITA & $\begin{array}{l}\text { Andreas } \\
\text { Husted }\end{array}$ \\
$\begin{array}{l}\text { Sygehus Lillebæalt, } \\
\text { Kolding Sygehus }\end{array}$ & Kolding & $\begin{array}{l}\text { Bedøvelse og } \\
\text { Intensiv }\end{array}$ & $\begin{array}{l}\text { Bård E. S. } \\
\text { Dahle }\end{array}$ \\
$\begin{array}{lll}\text { Aarhus University } \\
\text { Hospital }\end{array}$ & Aarhus & Intensive Care East & $\begin{array}{l}\text { Christine } \\
\text { Cramer }\end{array}$ \\
$\begin{array}{l}\text { Viborg Regional } \\
\text { Hospital }\end{array}$ & Viborg & $\begin{array}{l}\text { Department of } \\
\text { Anaesthesiology } \\
\text { and Intensive Care }\end{array}$ & $\begin{array}{l}\text { Christoffer } \\
\text { Sølling }\end{array}$ \\
& &
\end{tabular}




\begin{tabular}{|c|c|c|c|}
\hline $\begin{array}{l}\text { Nordsjællands } \\
\text { Hospital, University } \\
\text { of Copenhagen }\end{array}$ & Hillerød & $\begin{array}{l}\text { Department of } \\
\text { Anaesthesiology and } \\
\text { Intensive Care }\end{array}$ & Dorthe Ørsnes \\
\hline $\begin{array}{l}\text { Regions Hospital } \\
\text { Herning }\end{array}$ & Herning & Intensiv Herning & $\begin{array}{l}\text { Jakob Edelberg } \\
\text { Thomsen }\end{array}$ \\
\hline Vejle & Vejle & A710 Vejle & $\begin{array}{l}\text { Jonas Juul } \\
\text { Pedersen }\end{array}$ \\
\hline $\begin{array}{l}\text { Regionshospital } \\
\text { Nordjylland Hjørring }\end{array}$ & Hjørring & Intensiv & $\begin{array}{l}\text { Mathilde } \\
\text { Hummelmose } \\
\text { Enevoldsen }\end{array}$ \\
\hline $\begin{array}{l}\text { Aarhus University } \\
\text { Hospital }\end{array}$ & Aarhus & Intensive Care North & $\begin{array}{l}\text { Thomas } \\
\text { Elkmann }\end{array}$ \\
\hline
\end{tabular}

\section{England}

\begin{tabular}{|c|c|c|c|}
\hline $\begin{array}{l}\text { Yeovil District } \\
\text { Hospital }\end{array}$ & Yeovil & $\begin{array}{l}\text { Intensive Care } \\
\text { Unit }\end{array}$ & $\begin{array}{l}\text { Agnieszka } \\
\text { Kubisz-Pudelko }\end{array}$ \\
\hline $\begin{array}{l}\text { Peterborough City } \\
\text { Hospital }\end{array}$ & Petersborough & Critical Care Unit & Alan Pope \\
\hline $\begin{array}{l}\text { Queen Elizabeth } \\
\text { Hospital }\end{array}$ & & $\begin{array}{l}\text { Critical Care } \\
\text { Queen Elizabeth } \\
\text { Hospital }\end{array}$ & Amy Collins \\
\hline $\begin{array}{l}\text { Croydon University } \\
\text { Hospital }\end{array}$ & Croydon & $\begin{array}{l}\text { Croydon } \\
\text { University } \\
\text { Hospital ITU }\end{array}$ & Ashok S. Raj \\
\hline $\begin{array}{l}\text { Royal Devon \& Exeter } \\
\text { NHS Foundation } \\
\text { Trust }\end{array}$ & Exeter & $\begin{array}{l}\text { Intensive Care } \\
\text { Unit }\end{array}$ & Carole Boulanger \\
\hline $\begin{array}{l}\text { South Tyneside } \\
\text { District Hospital }\end{array}$ & South Shields & ITU & Christian Frey \\
\hline Maidstone & Maidstone & $\begin{array}{l}\text { Intensive Care/ } \\
\text { High } \\
\text { Dependency }\end{array}$ & Ciaran Hart \\
\hline $\begin{array}{l}\text { University Hospital } \\
\text { Southampton }\end{array}$ & Southampton & $\begin{array}{l}\text { General Intensive } \\
\text { Care Unit }\end{array}$ & Clare Bolger \\
\hline $\begin{array}{l}\text { St George's University } \\
\text { Hospitals NHS } \\
\text { Foundation trust }\end{array}$ & London & $\begin{array}{l}\text { Cardiothoracic } \\
\text { Intensive Care } \\
\text { Unit (CTICU) }\end{array}$ & Dominic Spray \\
\hline $\begin{array}{l}\text { Norfolk and Norwich } \\
\text { University Hospital }\end{array}$ & Norwich & $\begin{array}{l}\text { Critical care } \\
\text { complex }\end{array}$ & Georgina Randell \\
\hline $\begin{array}{l}\text { Royal Free Hospital } \\
\text { NHS Foundation } \\
\text { Trust }\end{array}$ & London & ICU 4 & Helder Filipe \\
\hline $\begin{array}{l}\text { Royal Liverpool } \\
\text { University Hospital }\end{array}$ & Liverpool & Intensive care & $\begin{array}{l}\text { Ingeborg D } \\
\text { Welters }\end{array}$ \\
\hline $\begin{array}{l}\text { Royal Hampshire } \\
\text { County Hospital }\end{array}$ & Winchester & $I C U$ & Irina Grecu \\
\hline $\begin{array}{l}\text { St George's University } \\
\text { Hospitals NHS } \\
\text { Foundation trust }\end{array}$ & London & $\begin{array}{l}\text { Acute } \\
\text { Dependency } \\
\text { Unit }\end{array}$ & Jane Evans \\
\hline $\begin{array}{l}\text { Blackpool Victoria } \\
\text { Hospital }\end{array}$ & Blackpool & $\begin{array}{l}\text { General Critical } \\
\text { Care Unit }\end{array}$ & Jason Cupitt \\
\hline Worthing Hospital & Worthing & $I C U$ & Jenny Lord \\
\hline $\begin{array}{l}\text { James Cook } \\
\text { University Hospital }\end{array}$ & Midlesbrough & ICU 2 and 2 & Jeremy Henning \\
\hline
\end{tabular}

(Continued)

\begin{tabular}{|c|c|c|c|}
\hline $\begin{array}{l}\text { Tunbridge Wells } \\
\text { Hospital }\end{array}$ & Pembury & $\begin{array}{l}\text { Intensive care } \\
\text { unit }\end{array}$ & Joanne Jones \\
\hline $\begin{array}{l}\text { St George's University } \\
\text { Hospitals NHS } \\
\text { Foundation trust }\end{array}$ & London & $\begin{array}{l}\text { Neuro Intensive } \\
\text { Care }\end{array}$ & Jonathan Ball \\
\hline $\begin{array}{l}\text { James Paget } \\
\text { University Hospital }\end{array}$ & Norfolk & ICU/HDU & Julie North \\
\hline $\begin{array}{l}\text { Royal Papworth } \\
\text { Hospital NHS } \\
\text { Foundation Trust }\end{array}$ & Cambridge & ICU & Kiran Salaunkey \\
\hline $\begin{array}{l}\text { Royal Sussex County } \\
\text { Hospital }\end{array}$ & Brighton & Level 7 & $\begin{array}{l}\text { Laura Ortiz-Ruiz } \\
\text { De Gordoa }\end{array}$ \\
\hline Salisbury & Salisbury & Radnor & Louise Bell \\
\hline Royal Bolton Hospital & Bolton & $\begin{array}{l}\text { Royal Bolton } \\
\text { CRITICAL CARE }\end{array}$ & $\begin{array}{l}\text { Madhu } \\
\text { Balasubramaniam }\end{array}$ \\
\hline $\begin{array}{l}\text { Chelsea and } \\
\text { Westminster Hospital }\end{array}$ & London & $\begin{array}{l}\text { Adult Intensive } \\
\text { Care Unit }\end{array}$ & $\begin{array}{l}\text { Marcela } \\
\text { Vizcaychipi }\end{array}$ \\
\hline $\begin{array}{l}\text { Countess of Chester } \\
\text { Hospital }\end{array}$ & Chester & $\begin{array}{l}\text { Intensive Care } \\
\text { Unit }\end{array}$ & Maria Faulkner \\
\hline $\begin{array}{l}\text { Hampshire Hospitals } \\
\text { Foundation Trust }\end{array}$ & Basingstoke & $\begin{array}{l}\text { Basingstoke and } \\
\text { North Hampshire } \\
\text { Hospital }\end{array}$ & $\begin{array}{l}\text { McDonald } \\
\text { Mupudzi }\end{array}$ \\
\hline $\begin{array}{l}\text { Hinchingbrooke } \\
\text { Hospital }\end{array}$ & Huntingdon & Critical Care & $\begin{array}{l}\text { Megan Lea- } \\
\text { Hagerty }\end{array}$ \\
\hline Russells Hall Hospital & Dudley & $\begin{array}{l}\text { Intensive Care } \\
\text { Unit Russells Hall }\end{array}$ & Michael Reay \\
\hline $\begin{array}{l}\text { Royal Cornwall } \\
\text { Hospital Trust }\end{array}$ & Cornwall & Critical Care Unit & Michael Spivey \\
\hline $\begin{array}{l}\text { Northern Devon } \\
\text { Healthcare NHS Trust }\end{array}$ & Barnstaple & $\begin{array}{l}\text { North Devon } \\
\text { District Hospital }\end{array}$ & Nicholas Love \\
\hline $\begin{array}{l}\text { Chesterfield Royal } \\
\text { Hospital }\end{array}$ & Chesterfield & $\begin{array}{l}\text { Intensive Care } \\
\text { Unit }\end{array}$ & $\begin{array}{l}\text { Nick Spittle Nick } \\
\text { Spittle }\end{array}$ \\
\hline $\begin{array}{l}\text { Royal Bournemouth } \\
\text { Hospital }\end{array}$ & Bournemouth & $\begin{array}{l}\text { Bournemouth } \\
\text { Critical Care Unit }\end{array}$ & Nigel White \\
\hline Dorset County & Dorchester & ICU DCH & Patricia Williams \\
\hline $\begin{array}{l}\text { Surrey and Sussex } \\
\text { Healthcare NHS Trust }\end{array}$ & Redhill & $\begin{array}{l}\text { East Surrey } \\
\text { Hospital }\end{array}$ & Patrick Morgan \\
\hline Darent Valley & Dartford & ICU & Phillipa Wakefield \\
\hline $\begin{array}{l}\text { Royal Surrey County } \\
\text { Hospital }\end{array}$ & Guildford & Royal Surrey & Rachel Savine \\
\hline $\begin{array}{l}\text { Wirral University } \\
\text { Teaching Hospital }\end{array}$ & Birkenhead & Critical care & Reni Jacob \\
\hline $\begin{array}{l}\text { Musgrove Park } \\
\text { Hospital }\end{array}$ & Taunton & Critical care Unit & Richard Innes \\
\hline $\begin{array}{l}\text { Kent and Canterbury } \\
\text { Hospital }\end{array}$ & Canterbury & K\&C ITU & Ritoo Kapoor \\
\hline $\begin{array}{l}\text { West Suffolk NHS } \\
\text { Foundation Trust }\end{array}$ & $\begin{array}{l}\text { Bury St } \\
\text { Edmunds }\end{array}$ & Critical Care & Sally Humphreys \\
\hline QAH & Portsmouth & $\begin{array}{l}\text { Dept Critical } \\
\text { Care QAH } \\
\text { (DCCQ) }\end{array}$ & Steve Rose \\
\hline Whiston Hospital & Liverpool & Ward 4E & Susan Dowling \\
\hline $\begin{array}{l}\text { St George's University } \\
\text { Hospitals NHS } \\
\text { Foundation trust }\end{array}$ & London & $\begin{array}{l}\text { General Intensive } \\
\text { care }\end{array}$ & Susannah Leaver \\
\hline
\end{tabular}


(Continued)

\begin{tabular}{|c|c|c|c|}
\hline $\begin{array}{l}\text { North Tees University } \\
\text { Hospital }\end{array}$ & $\begin{array}{l}\text { Stockton on } \\
\text { Tees }\end{array}$ & Critical Care Unit & $\begin{array}{l}\text { Tarkeshwari } \\
\text { Mane }\end{array}$ \\
\hline $\begin{array}{l}\text { Bradford Teaching } \\
\text { Hospitals NHS } \\
\text { Foundation Trust }\end{array}$ & Bradford & $\begin{array}{l}\text { Bradford Royal } \\
\text { Infirmary }\end{array}$ & Tom Lawton \\
\hline $\begin{array}{l}\text { Medway Maritime } \\
\text { Hospital }\end{array}$ & Medway & $\begin{array}{l}\text { Adult Intensive } \\
\text { Care Unit }\end{array}$ & Vongayi Ogbeide \\
\hline $\begin{array}{l}\text { University Hospital } \\
\text { Lewisham }\end{array}$ & Lewisham & $\begin{array}{l}\text { ICU/HDU } \\
\text { Lewisham }\end{array}$ & Waqas Khaliq \\
\hline St Richards Hospital & Chichester & Itchenor & Yolanda Baird \\
\hline
\end{tabular}

\section{France}

\begin{tabular}{|c|c|c|c|}
\hline $\begin{array}{l}\text { CH Francois } \\
\text { Mitterand }\end{array}$ & Pau & Reanimation polyvalente & $\begin{array}{l}\text { Antoine } \\
\text { Romen }\end{array}$ \\
\hline $\begin{array}{l}\text { Hôpital Privé } \\
\text { Claude Galien }\end{array}$ & $\begin{array}{l}\text { Quincy } \\
\text { sous } \\
\text { Sénart }\end{array}$ & Polyvalente & $\begin{array}{l}\text { Arnaud } \\
\text { Galbois }\end{array}$ \\
\hline Saint Antoine & Paris & $\begin{array}{l}\text { Medecine Intensive } \\
\text { Reanimation }\end{array}$ & $\begin{array}{l}\text { Bertrand } \\
\text { Guidet }\end{array}$ \\
\hline $\begin{array}{l}\text { Germon and } \\
\text { Gauthier }\end{array}$ & Béthune & $\begin{array}{l}\text { Médecine Intensive } \\
\text { Réanimation }\end{array}$ & $\begin{array}{l}\text { Christophe } \\
\text { Vinsonneau }\end{array}$ \\
\hline $\begin{array}{l}\text { Hôpital Ambroise } \\
\text { Paré }\end{array}$ & $\begin{array}{l}\text { Boulogne } \\
\text { Billancourt }\end{array}$ & $\begin{array}{l}\text { Medecine Intensive } \\
\text { Reanimation }\end{array}$ & $\begin{array}{l}\text { Cyril } \\
\text { Charron }\end{array}$ \\
\hline CH Dr. Schaffner & Lens & Reanimation polyvalente & $\begin{array}{l}\text { Didier } \\
\text { Thevenin }\end{array}$ \\
\hline $\begin{array}{l}\text { Hopital Européen } \\
\text { Georges Pompidou }\end{array}$ & Paris & $\begin{array}{l}\text { Médecine Intensive } \\
\text { Réanimation }\end{array}$ & $\begin{array}{l}\text { Emmanuel } \\
\text { Guerot }\end{array}$ \\
\hline CHU de Besançon & Besançon & $\begin{array}{l}\text { Département de } \\
\text { Anesthésie Réanimation } \\
\text { Chirurgicale }\end{array}$ & $\begin{array}{l}\text { Guillaume } \\
\text { Besch }\end{array}$ \\
\hline Hôpital Cochin & Paris & $\begin{array}{l}\text { Médecine Intensive } \\
\text { Réanimation }\end{array}$ & $\begin{array}{l}\text { Guillaume } \\
\text { Savary }\end{array}$ \\
\hline Victor Dupouy & Argenteuil & $\begin{array}{l}\text { Service de Réanimation } \\
\text { Polyvalente et USC }\end{array}$ & $\begin{array}{l}\text { Hervé } \\
\text { Mentec }\end{array}$ \\
\hline $\begin{array}{l}\text { Centre Hospitalier } \\
\text { Général }\end{array}$ & Cambrai & Réanimation polyvalente & $\begin{array}{l}\text { Jean-Luc } \\
\text { Chagnon }\end{array}$ \\
\hline $\begin{array}{l}\text { Dieppe General } \\
\text { Hospital }\end{array}$ & Dieppe & $\begin{array}{l}\text { Médecine Intensive } \\
\text { Réanimation }\end{array}$ & $\begin{array}{l}\text { Jean- } \\
\text { Philippe } \\
\text { Rigaud }\end{array}$ \\
\hline $\begin{array}{l}\text { CHU Dijon } \\
\text { Bourgogne }\end{array}$ & Dijon & $\begin{array}{l}\text { Medecine intensive- } \\
\text { Réanimation }\end{array}$ & $\begin{array}{l}\text { Jean-Pierre } \\
\text { Quenot }\end{array}$ \\
\hline $\mathrm{CH}$ Bigorre & Tarbes & $\begin{array}{l}\text { service de réanimation } \\
\text { polyvalente }\end{array}$ & $\begin{array}{l}\text { Jeremy } \\
\text { Castanera }\end{array}$ \\
\hline $\begin{array}{l}\mathrm{CH} \text { de Charleville- } \\
\text { Mézières }\end{array}$ & $\begin{array}{l}\text { Charleville- } \\
\text { Mezieres }\end{array}$ & $\begin{array}{l}\text { Medecine Intensive } \\
\text { Reanimation }\end{array}$ & $\begin{array}{l}\text { Jérémy } \\
\text { Rosman }\end{array}$ \\
\hline CHU Amiens & Amiens & Reanimaiton medicale & $\begin{array}{l}\text { Julien } \\
\text { Maizel }\end{array}$ \\
\hline $\begin{array}{l}\text { Groupe Hospitalier } \\
\text { Paris Saint Joseph }\end{array}$ & Paris & Réanimation polyvalente & $\begin{array}{l}\text { Kelly } \\
\text { Tiercelet }\end{array}$ \\
\hline CHU de Besançon & Besancon & Réanimation Médicale & $\begin{array}{l}\text { Lucie } \\
\text { Vettoretti }\end{array}$ \\
\hline
\end{tabular}

\begin{tabular}{|c|c|c|c|}
\hline CH DAX & Dax & Réanimation polyvalente & $\begin{array}{l}\text { Maud } \\
\text { Mousset } \\
\text { Hovaere }\end{array}$ \\
\hline Louis Mourier & Colombes & $\begin{array}{l}\text { Réanimation médico- } \\
\text { chirurgicale }\end{array}$ & $\begin{array}{l}\text { Messika } \\
\text { Messika }\end{array}$ \\
\hline Tenon & Paris & $\begin{array}{l}\text { Service de Réanimation } \\
\text { Médico Chirurgicale }\end{array}$ & $\begin{array}{l}\text { Michel } \\
\text { Djibré }\end{array}$ \\
\hline $\begin{array}{l}\text { Groupe Hospitalier } \\
\text { Sud lle de France }\end{array}$ & Melun & $\begin{array}{l}\text { Département de médecine } \\
\text { intensive }\end{array}$ & $\begin{array}{l}\text { Nathalie } \\
\text { Rolin }\end{array}$ \\
\hline $\begin{array}{l}\text { Clinique Du } \\
\text { Millenaire }\end{array}$ & Montpellier & $\begin{array}{l}\text { Reanimation Chirurugicale } \\
\text { II et III }\end{array}$ & $\begin{array}{l}\text { Philippe } \\
\text { Burtin }\end{array}$ \\
\hline Marne La Vallee & Jossigny & Reanimation Polyvalente & $\begin{array}{l}\text { Pierre } \\
\text { Garcon }\end{array}$ \\
\hline CHU Lille & Lille & Critical Care Center & Saad Nseir \\
\hline CHU de Caen & Caen & $\begin{array}{l}\text { Service de Réanimation } \\
\text { Médicale }\end{array}$ & $\begin{array}{l}\text { Xavier } \\
\text { Valette }\end{array}$ \\
\hline
\end{tabular}

\section{Germany}

\begin{tabular}{|c|c|c|c|}
\hline $\begin{array}{l}\text { Klinikum rechts der Isar } \\
\text { TU München }\end{array}$ & München & $\begin{array}{l}\text { Toxikologische } \\
\text { Intensivstation }\end{array}$ & $\begin{array}{l}\text { Christian } \\
\text { Rabe }\end{array}$ \\
\hline University Hospital Ulm & Ulm & $\begin{array}{l}\text { Anesthesiologic } \\
\text { Intensive Care } \\
\text { Department }\end{array}$ & $\begin{array}{l}\text { Eberhard } \\
\text { Barth }\end{array}$ \\
\hline $\begin{array}{l}\text { Katholisches Krankenhaus } \\
\text { St. Johann Nepomuk }\end{array}$ & Erfurt & $\begin{array}{l}\text { Klinik für Innere } \\
\text { Medizin II/ } \\
\text { Kardiologie } \\
\text { und Internistische } \\
\text { Intensivmedizin }\end{array}$ & $\begin{array}{l}\text { Henning } \\
\text { Ebelt }\end{array}$ \\
\hline $\begin{array}{l}\text { Klinikum rechts der Isar, } \\
\text { School of Medicine, } \\
\text { Technical University } \\
\text { of Munich }\end{array}$ & München & $\begin{array}{l}\text { Intensivstation } \\
\text { IS } 2 / L 2 a\end{array}$ & $\begin{array}{l}\text { Kristina } \\
\text { Fuest }\end{array}$ \\
\hline $\begin{array}{l}\text { Jena University Hospital, } \\
\text { Department of Internal } \\
\text { Medicine I }\end{array}$ & Jena & $\begin{array}{l}\text { Internistische } \\
\text { Intensivstation }\end{array}$ & $\begin{array}{l}\text { Marcus } \\
\text { Franz }\end{array}$ \\
\hline $\begin{array}{l}\text { West German Heart } \\
\text { and Vascular Center } \\
\text { Essen (WHGZ) }\end{array}$ & Essen & INTK & $\begin{array}{l}\text { Michael } \\
\text { Horacek }\end{array}$ \\
\hline $\begin{array}{l}\text { Universitätsmedizin der } \\
\text { Johannes Gutenberg- } \\
\text { Universität Mainz }\end{array}$ & Mainz & $\begin{array}{l}\text { Anästhesie- } \\
\text { Intensivstation }\end{array}$ & $\begin{array}{l}\text { Michael } \\
\text { Schuster }\end{array}$ \\
\hline $\begin{array}{l}\text { University Hospital } \\
\text { Frankfurt }\end{array}$ & $\begin{array}{l}\text { Frankfurt } \\
\text { am Main }\end{array}$ & $\begin{array}{l}\text { Department of } \\
\text { Anaesthesiology, } \\
\text { Intensive Care } \\
\text { Medicine and } \\
\text { Pain Therapy }\end{array}$ & $\begin{array}{l}\text { Patrick } \\
\text { Meybohm }\end{array}$ \\
\hline $\begin{array}{l}\text { University Hospital } \\
\text { Düsseldorf }\end{array}$ & Düsseldorf & $\mathrm{Ml} 1 / 2$ & $\begin{array}{l}\text { Raphael } \\
\text { Romano } \\
\text { Bruno }\end{array}$ \\
\hline $\begin{array}{l}\text { Robert-Bosch- } \\
\text { Krankenhaus }\end{array}$ & Stuttgart & 1D & $\begin{array}{l}\text { Sebastian } \\
\text { Allgäuer }\end{array}$ \\
\hline $\begin{array}{l}\text { Heidelberg University } \\
\text { Hospital }\end{array}$ & Heidelberg & Station 13 IOPIS & $\begin{array}{l}\text { Simon } \\
\text { Dubler }\end{array}$ \\
\hline
\end{tabular}




\section{(Continued)}

\begin{tabular}{|c|c|c|c|}
\hline $\begin{array}{l}\text { Klinikum rechts der Isar, } \\
\text { School of Medicine, } \\
\text { Technical University of } \\
\text { Munich }\end{array}$ & München & $\begin{array}{l}\text { Intensivstation } \\
\text { IS1 / M2b }\end{array}$ & $\begin{array}{l}\text { Stefan J } \\
\text { Schaller }\end{array}$ \\
\hline $\begin{array}{l}\text { University Hospital } \\
\text { Leipzig }\end{array}$ & Leipzig & $\begin{array}{l}\text { Department of } \\
\text { Anesthesiology and } \\
\text { Intensive Care Medicine }\end{array}$ & $\begin{array}{l}\text { Stefan } \\
\text { Schering }\end{array}$ \\
\hline St Vincenz Hospital & $\begin{array}{l}\text { Limburg/ } \\
\text { Lahn }\end{array}$ & Intensive care unit & $\begin{array}{l}\text { Stephan } \\
\text { Steiner }\end{array}$ \\
\hline Hannover Medical School & Hannover & 44 & $\begin{array}{l}\text { Thorben } \\
\text { Dieck }\end{array}$ \\
\hline $\begin{array}{l}\text { Universitätsklinikum } \\
\text { Knappschaftskrankenhaus } \\
\text { Bochum }\end{array}$ & Bochum & Operative IBA & $\begin{array}{l}\text { Tim } \\
\text { Rahmel }\end{array}$ \\
\hline $\begin{array}{l}\text { Universitätsklinikum } \\
\text { Schleswig-Holstein }\end{array}$ & Lübeck & IKI 12a & $\begin{array}{l}\text { Tobias } \\
\text { Graf }\end{array}$ \\
\hline
\end{tabular}

Greece

\begin{tabular}{|c|c|c|c|}
\hline Asklepieio Voulas & Athens & ICU & Anastasia Koutsikou \\
\hline Xanthi General Hospital & Xanthi & Xanthi ICU & Aristeidis Vakalos \\
\hline $\begin{array}{l}\text { Sismanoglio - Amallia } \\
\text { Fleming G. H }\end{array}$ & $\begin{array}{l}\text { Marousi - } \\
\text { Athens } \\
\text { Attika }\end{array}$ & Sismanoglio & Bogdan Raitsiou \\
\hline $\begin{array}{l}\text { General Hospital Agios } \\
\text { Pavlos }\end{array}$ & Thessaloniki & $\begin{array}{l}\text { ICU Agios } \\
\text { Pavlos }\end{array}$ & Elli Niki Flioni \\
\hline $\begin{array}{l}\text { General Hospital of } \\
\text { Larissa }\end{array}$ & Larissa & General ICU & Evangelia Neou \\
\hline Lamia General Hospita & Lamia & Lamia ICU & Fotios Tsimpoukas \\
\hline $\begin{array}{l}\text { University Hospital of } \\
\text { loannina }\end{array}$ & loannina & $\begin{array}{l}\text { Intensive Care } \\
\text { Unit }\end{array}$ & $\begin{array}{l}\text { Georgios } \\
\text { Papathanakos }\end{array}$ \\
\hline $\begin{array}{l}\text { General Hospital of } \\
\text { Athens Korgialeneio } \\
\text { Mbenakeio Red Cross }\end{array}$ & Athens & $\mathrm{ICU}$ & Giorgos Marinakis \\
\hline $\begin{array}{l}\text { General Hospital of } \\
\text { Eleusis Thriassio }\end{array}$ & Eleusis & ICU Latsio & $\begin{array}{l}\text { loannis } \\
\text { Koutsodimitropoulos }\end{array}$ \\
\hline $\begin{array}{l}\text { KONSTANTOPOULEION } \\
\text { GEN. HOSPITAL }\end{array}$ & Athens & General ICU & $\begin{array}{l}\text { Kounougeri } \\
\text { Aikaterini }\end{array}$ \\
\hline Sotiria Hospital & Athens & $\begin{array}{l}\text { ICU 1st } \\
\text { Department } \\
\text { of Pulmonary } \\
\text { Medicine } \\
\text { Athens } \\
\text { Medical } \\
\text { School, } \\
\text { National and } \\
\text { Kapodistrian } \\
\text { University of } \\
\text { Athens }\end{array}$ & Nikoletta Rovina \\
\hline $\begin{array}{l}\text { General Hospital of } \\
\text { Patra }\end{array}$ & Achaia & $\mathrm{ICU}$ & Stylliani Kourelea \\
\hline $\begin{array}{l}\text { G Gennimatas Hospital } \\
\text { of Thessaloniki }\end{array}$ & Thessaloniki & $\begin{array}{l}\text { ICU G } \\
\text { GENNIMATAS }\end{array}$ & $\begin{array}{l}\text { Polychronis } \\
\text { Tasioudis }\end{array}$ \\
\hline Agioi Anargiroi Hospital & Athens & General ICU & Vasiiios Zidianakis \\
\hline Theagenio & Theassaloniki & $\begin{array}{l}\text { Meth } \\
\text { Theagenio }\end{array}$ & Vryza Konstantinia \\
\hline
\end{tabular}

(Continued)

\begin{tabular}{llll}
\hline University General & Thessaloniki & Metha & Zoi Aidoni \\
Hospital Ahepa & & \\
\hline
\end{tabular}

Ireland

\begin{tabular}{lcll}
\hline $\begin{array}{l}\text { Mater Misericordiae } \\
\text { University Hospital }\end{array}$ & Dublin & $\begin{array}{l}\text { Department of Critical } \\
\text { Care Medicine }\end{array}$ & Brian Marsh \\
\hline $\begin{array}{l}\text { University Hospital } \\
\text { Limerick }\end{array}$ & Limerick & UHL ICU & $\begin{array}{l}\text { Catherine } \\
\text { Motherway }\end{array}$ \\
$\begin{array}{l}\text { University Hospital } \\
\text { Galway }\end{array}$ & Galway & General ICU & $\begin{array}{l}\text { Chris Read } \\
\text { St James's Hospital }\end{array}$ \\
& Dublin & ICU & $\begin{array}{l}\text { Ignacio } \\
\text { Martin- } \\
\text { Loeches }\end{array}$ \\
& & & \\
& & &
\end{tabular}

Italy

\begin{tabular}{llll}
\hline $\begin{array}{l}\text { Arnas Ospedale Civico } \\
\text { De Christina Benfratelli }\end{array}$ & Palermo & $\begin{array}{l}\text { Terapia Intensiva } \\
\text { Polivalente Con Trauma } \\
\text { Center }\end{array}$ & $\begin{array}{l}\text { Andrea } \\
\text { Neville } \\
\text { Cracchiolo }\end{array}$ \\
$\begin{array}{l}\text { Istituto Ortopedico } \\
\text { Rizzoli }\end{array}$ & Bologna & TIPO & Aristide \\
San Giuseppe & Empoli & Terapia Intensiva & Italo Calamai \\
$\begin{array}{l}\text { Humanitas Reseach } \\
\text { Hospital }\end{array}$ & Milan & General ICU & Stefania \\
& & & Brusa \\
\hline
\end{tabular}

\section{Libya}

\begin{tabular}{llll}
\hline Al-Zawia University Hospital & Al-Zawia & ICU & Ahmed Elhadi \\
Alkhums Hospital & Alkhums & ICU & Ahmed Tarek \\
Elkhadra Hospital & Tripoli & ICU & Ala Khaled \\
Abo Selim Trauma Hospital & Tripoli & ICU & Hazem Ahmed \\
Tripoli Medical Center & Tripoli & CCU & Wesal Ali Belkhair \\
& & & \\
\hline
\end{tabular}

\section{Netherland}

\begin{tabular}{|c|c|c|c|}
\hline $\begin{array}{l}\text { Medisch Spectrum } \\
\text { Twente }\end{array}$ & Enschede & $\begin{array}{l}\text { Intensive Care } \\
\text { Center }\end{array}$ & $\begin{array}{l}\text { Alexander D. } \\
\text { Cornet }\end{array}$ \\
\hline Erasmus Medical Center & Rotterdam & ICU adults & $\begin{array}{l}\text { Diederik } \\
\text { Gommers }\end{array}$ \\
\hline UMC Utrecht & Utrecht & ICU departement & $\begin{array}{l}\text { Dylan de } \\
\text { Lange }\end{array}$ \\
\hline $\begin{array}{l}\text { Albert Schweitzer } \\
\text { Ziekenhuis }\end{array}$ & Dordrecht & ICU asz & $\begin{array}{l}\text { Eva van } \\
\text { Boven }\end{array}$ \\
\hline Isala Hospital & Zwolle & Intensive Care & $\begin{array}{l}\text { Jasper } \\
\text { Haringman }\end{array}$ \\
\hline Diakonessenhuis Utrecht & Utrecht & Intensive care & Lenneke Haas \\
\hline Haga Ziekenhuis & The Hague & $\mathrm{ICU}$ & \\
\hline
\end{tabular}


(Continued)

\begin{tabular}{|c|c|c|c|}
\hline & & & $\begin{array}{l}\text { Lettie van } \\
\text { den Berg }\end{array}$ \\
\hline $\begin{array}{l}\text { Canisius Wilhelmina } \\
\text { Ziekenhuis }\end{array}$ & Nijmegen & C38 & Oscar Hoiting \\
\hline Jeroen Bosch Ziekenhuis & Den Bosch & IC JBZ & $\begin{array}{l}\text { Peter de } \\
\text { Jager }\end{array}$ \\
\hline $\begin{array}{l}\text { Medical Centre } \\
\text { Leeuwarden }\end{array}$ & Leeuwarden & $\begin{array}{l}\text { Department of } \\
\text { Intensive Care }\end{array}$ & $\begin{array}{l}\text { Rik T. } \\
\text { Gerritsen }\end{array}$ \\
\hline $\begin{array}{l}\text { Zuyderland Medical } \\
\text { Center }\end{array}$ & Heerlen & Zuyderland Heerlen & $\begin{array}{l}\text { Tom } \\
\text { Dormans }\end{array}$ \\
\hline $\begin{array}{l}\text { University Medical } \\
\text { Center Groningen }\end{array}$ & Groningen & $\begin{array}{l}\text { Department of } \\
\text { Critical Care }\end{array}$ & $\begin{array}{l}\text { Willem } \\
\text { Dieperink }\end{array}$ \\
\hline
\end{tabular}

\section{Norway}

\begin{tabular}{|c|c|c|c|}
\hline $\begin{array}{l}\text { Førde Central } \\
\text { Hospital }\end{array}$ & Førde & $\begin{array}{l}\text { Department of } \\
\text { Emergency Medicine } \\
\text { and Inensive Care }\end{array}$ & $\begin{array}{l}\text { Alena } \\
\text { Breidablik } \\
\text { Alena } \\
\text { Breidablik }\end{array}$ \\
\hline Kongsberg & Kongsberg & Intensivavdelingen & $\begin{array}{l}\text { Anita } \\
\text { Slapgard }\end{array}$ \\
\hline Sykehuset Østfold & Grålum & Intensiv & $\begin{array}{l}\text { Anne-Karin } \\
\text { Rime }\end{array}$ \\
\hline Sykehuset Telemark & Skien & Intensiv Skien & $\begin{array}{l}\text { Bente } \\
\text { Jannestad }\end{array}$ \\
\hline $\begin{array}{l}\text { Haukeland University } \\
\text { Hospital }\end{array}$ & Bergen & General ICU & Britt Sjøbøe \\
\hline Ålesund & Ålesund & Medisinsk intensiv & Eva Rice \\
\hline Ålesund hospital & Ålesund & $\begin{array}{l}\text { Dept. Anesthesia and } \\
\text { Intensive Care, Surgical } \\
\text { ICU }\end{array}$ & $\begin{array}{l}\text { Finn } \mathrm{H} \text {. } \\
\text { Andersen }\end{array}$ \\
\hline $\begin{array}{l}\text { Kristiansund sykehus } \\
\text { Helse Møre og } \\
\text { Romsdal HF }\end{array}$ & $\begin{array}{l}\text { Kristiansund } \\
\mathrm{N}\end{array}$ & Intensiv Kristiansund & $\begin{array}{l}\text { Hans Frank } \\
\text { Strietzel }\end{array}$ \\
\hline Namsos Sykehus & Namsos & Intensivavdeling & $\begin{array}{l}\text { Jan Peter } \\
\text { Jensen }\end{array}$ \\
\hline $\begin{array}{l}\text { Haukeland University } \\
\text { Hospital }\end{array}$ & Bergen & $\begin{array}{l}\text { Medisinsk intensiv og } \\
\text { overvåkning (MIO) }\end{array}$ & $\begin{array}{l}\text { Jørund } \\
\text { Langørgen }\end{array}$ \\
\hline $\begin{array}{l}\text { Oslo University } \\
\text { Hospital }\end{array}$ & Oslo & $\begin{array}{l}\text { Intensive Care section } \\
\text { Ullevaal }\end{array}$ & Kirsti Tøien \\
\hline $\begin{array}{l}\text { Stavanger University } \\
\text { Hospital }\end{array}$ & Stavanger & $\begin{array}{l}\text { Department of } \\
\text { Intensive Care }\end{array}$ & $\begin{array}{l}\text { Kristian } \\
\text { Strand }\end{array}$ \\
\hline Haugesund sjukehus & Haugesund & Intensivavdelingen & Michael Hahn \\
\hline $\begin{array}{l}\text { St Olavs University } \\
\text { Hospital }\end{array}$ & Oslo & Hovedintensiv & Pål Klepstad \\
\hline
\end{tabular}

Poland

\begin{tabular}{llll}
\hline $\begin{array}{l}\text { Szpital Wojewódzki w } \\
\text { Bełchatowie }\end{array}$ & Bełchatów & $\begin{array}{l}\text { Oddział Intensywnej } \\
\text { Terapii }\end{array}$ & $\begin{array}{l}\text { Aleksandra } \\
\text { Biernacka }\end{array}$ \\
\hline $\begin{array}{l}\text { Heliodor Swiecicki } \\
\text { Clinical Hospital at the }\end{array}$ & Poznań & $\begin{array}{l}\text { Anaesthesiology } \\
\text { intensive care and pain }\end{array}$ & Anna Kluzik \\
$\begin{array}{l}\text { Karol Marcinkowski } \\
\text { Medical University in }\end{array}$ & & $\begin{array}{l}\text { treatment Department } \\
\text { Poztint }\end{array}$ & \\
& & &
\end{tabular}

Medical University in Poznan
(Continued)

\begin{tabular}{|c|c|c|c|}
\hline $\begin{array}{l}\text { Szpital Wojewódzki w } \\
\text { Bełchatowie }\end{array}$ & Bełchatów & $\begin{array}{l}\text { Oddział Intensywnej } \\
\text { Terapii }\end{array}$ & $\begin{array}{l}\text { Aleksandra } \\
\text { Biernacka }\end{array}$ \\
\hline $\begin{array}{l}\text { University Hospital in } \\
\text { Zielona Góra }\end{array}$ & $\begin{array}{l}\text { Zielona } \\
\text { Góra }\end{array}$ & $\begin{array}{l}\text { Clinical Department od } \\
\text { Anesthesiology and } \\
\text { Intensiv Care }\end{array}$ & $\begin{array}{l}\text { Bartosz } \\
\text { Kudlinski }\end{array}$ \\
\hline $\begin{array}{l}\text { Regional Teaching } \\
\text { Hospital }\end{array}$ & $\begin{array}{l}\text { Bielsko- } \\
\text { Biała }\end{array}$ & $\begin{array}{l}\text { Department of } \\
\text { Anaesthesiology and } \\
\text { Intensive Care }\end{array}$ & $\begin{array}{l}\text { Dariusz } \\
\text { Maciejewski }\end{array}$ \\
\hline $\begin{array}{l}\text { St. John Grande } \\
\text { Hospital }\end{array}$ & Kraków & $\begin{array}{l}\text { Oddział Anestezjologii i } \\
\text { Intensywnej Terapii }\end{array}$ & $\begin{array}{l}\text { Dorota } \\
\text { Studzińska }\end{array}$ \\
\hline $\begin{array}{l}\text { The John Paul II } \\
\text { Hospital }\end{array}$ & Krakow & $\begin{array}{l}\text { Department of } \\
\text { Anesthesiology and } \\
\text { Intensive Care }\end{array}$ & $\begin{array}{l}\text { Hubert } \\
\text { Hymczak }\end{array}$ \\
\hline $\begin{array}{l}\text { Uniwersyteckie } \\
\text { Centrum Kliniczne w } \\
\text { Gdańsku }\end{array}$ & Gdańsk & $\begin{array}{l}\text { Klinika Anestezjologii i } \\
\text { Intensywnej Terapii }\end{array}$ & $\begin{array}{l}\text { Jan } \\
\text { Stefaniak }\end{array}$ \\
\hline $\begin{array}{l}\text { Pomeranian Medical } \\
\text { University }\end{array}$ & Szczecin & $\begin{array}{l}\text { Department of } \\
\text { Anesthesiology and } \\
\text { Intensive Care }\end{array}$ & $\begin{array}{l}\text { Joanna } \\
\text { Solek- } \\
\text { Pastuszka }\end{array}$ \\
\hline $\begin{array}{l}\text { University Hospital in } \\
\text { Cracow }\end{array}$ & Kraków & $\begin{array}{l}\text { Anaesthesiology and } \\
\text { Intensive Care Unit No.1 }\end{array}$ & $\begin{array}{l}\text { Joanna } \\
\text { Zorska }\end{array}$ \\
\hline $\begin{array}{l}\text { Regionalne Centrum } \\
\text { Zdrowia w Lubinie }\end{array}$ & Lubin & $\begin{array}{l}\text { Oddział Anestezjologii i } \\
\text { Intensywnej Terapii }\end{array}$ & $\begin{array}{l}\text { Katarzyna } \\
\text { Cwyl }\end{array}$ \\
\hline $\begin{array}{l}\text { University Clinical } \\
\text { Center Katowice }\end{array}$ & Katowice & $\begin{array}{l}\text { Department of } \\
\text { Anaesthesiology and } \\
\text { Intensive Care - School } \\
\text { of Medicine in Katowice, } \\
\text { Medical University of } \\
\text { Silesia }\end{array}$ & $\begin{array}{l}\text { Lukasz J. } \\
\text { Krzych }\end{array}$ \\
\hline Teching Hospital No 2 & Szczecin & $\begin{array}{l}\text { Department } \\
\text { Anaesthesiology } \\
\text { Intensive Therapy and } \\
\text { Acute Poisoning }\end{array}$ & $\begin{array}{l}\text { Maciej } \\
\text { Zukowski }\end{array}$ \\
\hline $\begin{array}{l}\text { 4th Military Hospital in } \\
\text { Wrocław }\end{array}$ & Wrocław & $\begin{array}{l}\text { Anesthesia and Intensive } \\
\text { Care Unit }\end{array}$ & $\begin{array}{l}\text { Małgorzata } \\
\text { Lipińska- } \\
\text { Gediga }\end{array}$ \\
\hline Centrum Chorób Płuc & Łódź & $\begin{array}{l}\text { Oddział Anestezjologii i } \\
\text { Intensywnej Terapii }\end{array}$ & $\begin{array}{l}\text { Marek } \\
\text { Pietruszko }\end{array}$ \\
\hline $\begin{array}{l}\text { The Dr. Wł. Biegański } \\
\text { Regional Specialist } \\
\text { Hospital in Łódź }\end{array}$ & Łódź & $\begin{array}{l}\text { Department of } \\
\text { Anaesthesiology and } \\
\text { Intensive Therapy - } \\
\text { Centre for Artificial } \\
\text { Extracorporeal Kidney } \\
\text { and Liver Support }\end{array}$ & $\begin{array}{l}\text { Mariusz } \\
\text { Piechota }\end{array}$ \\
\hline $\begin{array}{l}\text { Central Clinical } \\
\text { Hospital CKD - } \\
\text { University Medical } \\
\text { College in Lodz }\end{array}$ & Lodz & $\begin{array}{l}\text { Anaesthesia and } \\
\text { Intensive Care Clinic }\end{array}$ & Marta Serwa \\
\hline $\begin{array}{l}\text { First Independent } \\
\text { Teaching Hospital No. } \\
1\end{array}$ & Lublin & $\begin{array}{l}\text { II Department of } \\
\text { Anesthesiology and } \\
\text { Intensive Care }\end{array}$ & $\begin{array}{l}\text { Miroslaw } \\
\text { Czuczwar }\end{array}$ \\
\hline $\begin{array}{l}\text { Krakowski Szpital } \\
\text { Specjalistyczny im. } \\
\text { Jana Pawła II }\end{array}$ & Kraków & $\begin{array}{l}\text { Thoracic Anaesthesia } \\
\text { and Respiratory ICU }\end{array}$ & $\begin{array}{l}\text { Mirosław } \\
\text { Ziętkiewicz }\end{array}$ \\
\hline $\begin{array}{l}\text { Wroclaw Medical } \\
\text { University }\end{array}$ & Wroclaw & $\begin{array}{l}\text { Department of } \\
\text { Anesthesiology and } \\
\text { Intensve Therapy }\end{array}$ & $\begin{array}{l}\text { Natalia } \\
\text { Kozera }\end{array}$ \\
\hline $\begin{array}{l}\text { Szpital św.Anny W } \\
\text { Miechowie }\end{array}$ & Miechów & $\begin{array}{l}\text { Oddział Anestezjologii i } \\
\text { Intensywnej Terapii }\end{array}$ & $\begin{array}{l}\text { Paweł } \\
\text { Nasiłowski }\end{array}$ \\
\hline
\end{tabular}


(Continued)

\begin{tabular}{|c|c|c|c|}
\hline $\begin{array}{l}\text { Szpital Wojewódzki w } \\
\text { Bełchatowie }\end{array}$ & Bełchatów & $\begin{array}{l}\text { Oddział Intensywnej } \\
\text { Terapii }\end{array}$ & $\begin{array}{l}\text { Aleksandra } \\
\text { Biernacka }\end{array}$ \\
\hline $\begin{array}{l}\text { University Hospital in } \\
\text { Krakow }\end{array}$ & Krakow & ICU Skawinska & $\begin{array}{l}\text { Paweł } \\
\text { Sendur }\end{array}$ \\
\hline $\begin{array}{l}\text { Infant Jesus Teaching } \\
\text { Hospital }\end{array}$ & Warsaw & $\begin{array}{l}\text { I Department of } \\
\text { Anaesthesiology and } \\
\text { Intensive Care }\end{array}$ & $\begin{array}{l}\text { Paweł } \\
\text { Zatorski }\end{array}$ \\
\hline $\begin{array}{l}\text { Regional Hospital in } \\
\text { Bialystok }\end{array}$ & Bialystok & $\begin{array}{l}\text { Department of } \\
\text { Anaesthesiology and } \\
\text { Intensive Care }\end{array}$ & Piotr Galkin \\
\hline $\begin{array}{l}\text { Opole University } \\
\text { Hospital }\end{array}$ & Opole & $\begin{array}{l}\text { Department of } \\
\text { Anesthesiology and } \\
\text { Intensive Care }\end{array}$ & $\begin{array}{l}\text { Ryszard } \\
\text { Gawda }\end{array}$ \\
\hline $\begin{array}{l}\text { University Hospital in } \\
\text { Bialystok }\end{array}$ & Bialystok & $\begin{array}{l}\text { Department of } \\
\text { Anaesthesiology and } \\
\text { Intensive Therapy }\end{array}$ & $\begin{array}{l}\text { Urszula } \\
\text { Kościuczuk }\end{array}$ \\
\hline $\begin{array}{l}\text { Dr Antoni Jurasz } \\
\text { University Hospital in } \\
\text { Bydgoszcz }\end{array}$ & Bydgoszcz & $\begin{array}{l}\text { Department of } \\
\text { Anesthesia and Critical } \\
\text { Care }\end{array}$ & $\begin{array}{l}\text { Waldemar } \\
\text { Cyrankiewicz }\end{array}$ \\
\hline $\begin{array}{l}\text { Saint Lucas Hospital, } \\
\text { Konskie }\end{array}$ & Konskie & $\begin{array}{l}\text { Intensive Care } \\
\text { Department }\end{array}$ & $\begin{array}{l}\text { Wojciech } \\
\text { Gola }\end{array}$ \\
\hline
\end{tabular}

\section{Portugal.}

\begin{tabular}{|c|c|c|c|}
\hline $\begin{array}{l}\text { Centro Hospitalar do } \\
\text { Porto }\end{array}$ & Oporto & $\begin{array}{l}\text { Serviço de Cuidados } \\
\text { Intensivos } 1\end{array}$ & $\begin{array}{l}\text { Alexandre } \\
\text { Fernandes } \\
\text { Pinto }\end{array}$ \\
\hline $\begin{array}{l}\text { Hospital S. José, CHULC } \\
\text { EPE }\end{array}$ & Lisboa & $\begin{array}{l}\text { UCI Neurocríticos e } \\
\text { Trauma }\end{array}$ & $\begin{array}{l}\text { Ana } \\
\text { Margarida } \\
\text { Fernandes }\end{array}$ \\
\hline $\begin{array}{l}\text { Hospital São Francisco } \\
\text { Xavier }\end{array}$ & Lisbon & $\begin{array}{l}\text { Unidade Cuidados } \\
\text { Intensivos Polivalente }\end{array}$ & $\begin{array}{l}\text { Ana Rita } \\
\text { Santos }\end{array}$ \\
\hline Hospital da Luz & Lisboa & UCl Hospital da Luz & Cristina Sousa \\
\hline Hospital de Viseu & Viseu & UCIP & Inês Barros \\
\hline $\begin{array}{l}\text { Hospital Professor Doutor } \\
\text { Fernando Fonseca EPE }\end{array}$ & Amadora & $\begin{array}{l}\text { Serviço de Medicina } \\
\text { Intensiva SMI }\end{array}$ & $\begin{array}{l}\text { Isabel Amorim } \\
\text { Ferreira }\end{array}$ \\
\hline $\begin{array}{l}\text { Hospital Garcia de Orta - } \\
\text { HGO }\end{array}$ & Almada & $\begin{array}{l}\text { Serviço de Medicina } \\
\text { Intensiva }\end{array}$ & $\begin{array}{l}\text { Jacobo } \\
\text { Bacariza } \\
\text { Blanco }\end{array}$ \\
\hline $\begin{array}{l}\text { Hospital São Bernardo - } \\
\text { CH Setúbal }\end{array}$ & Setúbal & $\begin{array}{l}\text { Serviço de Cuidados } \\
\text { Intensivos }\end{array}$ & $\begin{array}{l}\text { João Teles } \\
\text { Carvalho }\end{array}$ \\
\hline $\begin{array}{l}\text { Centro Hospitalar de Trás } \\
\text { Montes e Alto Douro }\end{array}$ & Vila Real & $\begin{array}{l}\text { Serviço de Medicina } \\
\text { Intensiva }\end{array}$ & Jose Maia \\
\hline Lusiadas Lisboa & Lisboa & UCl- Lusiadas & $\begin{array}{l}\text { Nuno } \\
\text { Candeias }\end{array}$ \\
\hline CHMT-Abrantes & Abrantes & SMl & Nuno Catorze \\
\hline \multicolumn{4}{|l|}{ Russia. } \\
\hline $\begin{array}{l}\text { Privolzhskiy District } \\
\text { Medical Center }\end{array}$ & $\begin{array}{l}\text { Nizhniy } \\
\text { Novgorod }\end{array}$ & $\begin{array}{l}\text { epartment of } \\
\text { hesthesiology and Intensi } \\
\text { are }\end{array}$ & $\begin{array}{l}\text { Vladislav } \\
\text { Belskiy }\end{array}$ \\
\hline
\end{tabular}

Spain.

\begin{tabular}{|c|c|c|c|}
\hline Hospital De Bellvitge & Barcelona & $\mathrm{UCl}$ & Africa Lores \\
\hline $\begin{array}{l}\text { Hospital General } \\
\text { Universitario de } \\
\text { Albacete }\end{array}$ & Albacete & UCI Polivalente & $\begin{array}{l}\text { Angela Prado } \\
\text { Mira }\end{array}$ \\
\hline $\begin{array}{l}\text { Hospital Clinic of } \\
\text { Barcelona }\end{array}$ & Barcelona & $\begin{array}{l}\text { Respiratory Intensive } \\
\text { Care Unit }\end{array}$ & Catia Cilloniz \\
\hline $\begin{array}{l}\text { Hospital Universitario } \\
\text { Río Hortega }\end{array}$ & Valladolid & $\begin{array}{l}\text { UVI Polivalente y } \\
\text { Coronaria }\end{array}$ & $\begin{array}{l}\text { David Perez- } \\
\text { Torres }\end{array}$ \\
\hline Universitario La Paz & Madrid & Surgical ICU & $\begin{array}{l}\text { Emilio } \\
\text { Maseda }\end{array}$ \\
\hline $\begin{array}{l}\text { General Universitario } \\
\text { de Castellón }\end{array}$ & Castellón & $\begin{array}{l}\text { Servicio de Medicina } \\
\text { Intensiva }\end{array}$ & $\begin{array}{l}\text { Enver } \\
\text { Rodriguez }\end{array}$ \\
\hline $\begin{array}{l}\text { Hospital Universitario } \\
\text { Río Hortega }\end{array}$ & Valladolid & $\begin{array}{l}\text { UVI Neurocríticos } \\
\text { Trauma y } \\
\text { Quemados }\end{array}$ & $\begin{array}{l}\text { Estefania } \\
\text { Prol-Silva }\end{array}$ \\
\hline $\begin{array}{l}\text { Hospital de Tortosa } \\
\text { Verge de la Cinta }\end{array}$ & Tortosa & $\begin{array}{l}\text { Servei de Medicina } \\
\text { Intensiva }\end{array}$ & $\begin{array}{l}\text { Gaspar } \\
\text { Eixarch }\end{array}$ \\
\hline Parc Taulí & Sabadell & Parc Taulí & $\begin{array}{l}\text { Gemma } \\
\text { Gomà }\end{array}$ \\
\hline $\begin{array}{l}\text { Clínico Universitario } \\
\text { de Valencia }\end{array}$ & Valencia & $\begin{array}{l}\text { Surgical Intensive } \\
\text { Care Unit }\end{array}$ & $\begin{array}{l}\text { Gerardo } \\
\text { Aguilar }\end{array}$ \\
\hline $\begin{array}{l}\text { Hospital Universitario } \\
\text { de Torrejon }\end{array}$ & $\begin{array}{l}\text { Torrejon de } \\
\text { Ardoz, Madrid }\end{array}$ & Intensive Care UNit & $\begin{array}{l}\text { Gonzalo } \\
\text { Navarro } \\
\text { Velasco }\end{array}$ \\
\hline $\begin{array}{l}\text { Hospital General de } \\
\text { Catalunya }\end{array}$ & Barcelona & HGC & $\begin{array}{l}\text { Marián } \\
\text { Irazábal } \\
\text { Jaimes }\end{array}$ \\
\hline $\begin{array}{l}\text { Hospital Universitario } \\
\text { Sagrado Corazon }\end{array}$ & Barcelona & Intensive Care Unit & $\begin{array}{l}\text { Mercedes } \\
\text { Ibarz } \\
\text { Villamayor }\end{array}$ \\
\hline Hospital reina Sofía & Murcia & Reina Sofía & $\begin{array}{l}\text { Noemí } \\
\text { Llamas } \\
\text { Fernández }\end{array}$ \\
\hline $\begin{array}{l}\text { Complejo Hospitalario } \\
\text { de Segovia }\end{array}$ & Segovia & ICU Segovia & $\begin{array}{l}\text { Patricia } \\
\text { Jimeno } \\
\text { Cubero }\end{array}$ \\
\hline $\begin{array}{l}\text { Universitario de } \\
\text { Getafe }\end{array}$ & Getafe & $\begin{array}{l}\text { Intensive Care and } \\
\text { Burn Unit }\end{array}$ & $\begin{array}{l}\text { Sonia López- } \\
\text { Cuenca }\end{array}$ \\
\hline $\begin{array}{l}\text { Germans Trias i Pujol } \\
\text { Hospital }\end{array}$ & Badalona & General ICU & $\begin{array}{l}\text { Teresa } \\
\text { Tomasa }\end{array}$ \\
\hline $\begin{array}{l}\text { Centralsjukhuset i } \\
\text { Karlstad }\end{array}$ & Karlstad & IVA & $\begin{array}{l}\text { Anders } \\
\text { Sjöqvist }\end{array}$ \\
\hline
\end{tabular}

Sweden.

\begin{tabular}{llll}
\hline Umeå University & Umeå & $\begin{array}{l}\text { Department of Surgical } \\
\text { and Perioperative Sciences, } \\
\text { Anestesiology and } \\
\text { Intensive Care Medicine }\end{array}$ & $\begin{array}{l}\text { Camilla } \\
\text { Brorsson }\end{array}$ \\
\hline Vrinnevisjukhuset & Norrköping & IVA Norrköping & $\begin{array}{l}\text { Fredrik } \\
\text { Schiöler }\end{array}$ \\
Sundsvall Hospital & Sundsvall & Sundsvall ICU & $\begin{array}{l}\text { Henrik } \\
\text { Westberg }\end{array}$ \\
Blekingesjukhuset & Karlskrona & Intensivvårdsavdelning 31 & $\begin{array}{l}\text { Jessica } \\
\text { Nauska }\end{array}$
\end{tabular}


(Continued)

\begin{tabular}{llll}
\hline Umeå University & Umeå & $\begin{array}{l}\text { Department of Surgical } \\
\text { and Perioperative Sciences, } \\
\text { Anestesiology and } \\
\text { Intensive Care Medicine }\end{array}$ & $\begin{array}{l}\text { Camilla } \\
\text { Brorsson }\end{array}$ \\
\hline Alingsås Lasarett & Alingsås & Intensivvårdsavdelningen & $\begin{array}{l}\text { Joakim } \\
\text { Sivik }\end{array}$ \\
Västervikssjukhus & Västervik & IVA Västervikssjukhus & $\begin{array}{l}\text { Johan } \\
\text { Berkius }\end{array}$ \\
$\begin{array}{l}\text { Sahlgrenska University } \\
\text { Hospital/ Område 3/ }\end{array}$ & Göteborg & IVA avd 227 & $\begin{array}{l}\text { Karin } \\
\text { Kölndals sjukhus }\end{array}$ \\
$\begin{array}{l}\text { Linköping University } \\
\text { Hospital }\end{array}$ & Linköping & ICU Linköping & $\begin{array}{l}\text { Thiringer } \\
\text { Linköping University }\end{array}$ \\
Hospital & Linköping & Cardiothoracic Intensive \\
& & Care Unit & Seer \\
& & & Sten \\
\hline
\end{tabular}

\section{Switzerland.}

\begin{tabular}{lcll}
\hline $\begin{array}{l}\text { Hopitaux Universitaires } \\
\text { de Genève }\end{array}$ & Geneva & Adult Intensive Care Unit & $\begin{array}{l}\text { Filippo } \\
\text { Boroli }\end{array}$ \\
\hline $\begin{array}{l}\text { University of Bern } \\
\text { Inselspital }\end{array}$ & Bern & $\begin{array}{l}\text { Department of Intensive } \\
\text { Care Medicine }\end{array}$ & $\begin{array}{l}\text { Joerg C. } \\
\text { Schefold }\end{array}$ \\
$\begin{array}{l}\text { Fribourg Hospital } \\
\text { Intensive Care Unit }\end{array}$ & $\begin{array}{l}\text { Leila } \\
\text { Hergafi }\end{array}$ \\
$\begin{array}{l}\text { Centre Hospitalier } \\
\text { Universitaire Vaudois }\end{array}$ & Lausanne & $\begin{array}{l}\text { Service de médecine } \\
\text { intensive adulte }\end{array}$ & $\begin{array}{l}\text { Philippe } \\
\text { Eckert }\end{array}$
\end{tabular}

Turkey.

\begin{tabular}{lcll}
\hline $\begin{array}{l}\text { Ordu University Training and Research } \\
\text { Hospital }\end{array}$ & $\begin{array}{c}\text { Ordu } \\
\text { General }\end{array}$ & Ismail \\
& & YIldiz \\
\hline
\end{tabular}

Ukraine.

\begin{tabular}{llll}
\hline $\begin{array}{l}\text { Dnipro Mechnikov Regional Clinical } \\
\text { Hospital }\end{array}$ & Dnipro & $\begin{array}{l}\text { Intensive Care } \\
\text { Unit of Polytrauma }\end{array}$ & $\begin{array}{l}\text { Ihor } \\
\text { Yovenko }\end{array}$ \\
\hline $\begin{array}{l}\text { European Wellness Academy, } \\
\text { Luhansk Regional Clinical Hospital }\end{array}$ & Luhansk & ICU 1 & Yuriy \\
$\begin{array}{llll}\text { European Wellness Academy, } \\
\text { Luhansk Regional Clinical Hospital }\end{array}$ & Luhansk ICU 2 & Nalapko \\
& & & Yuriy \\
& & & Nalapko
\end{tabular}

Wales.

$\overline{\text { Glan Clwyd Hospital Bodelwyddan Critical Care Richard Pugh }}$

\section{Financial disclosure statement}

No (industry) sponsorship has been received for this investigator-initiated study.

\section{Authors' contributions}

$B W, R R B$ and $C J$ analysed the data and wrote the first draft of the manuscript. $\mathrm{HF}$ and $\mathrm{BG}$ and $\mathrm{DL}$ contributed to statistical analysis and improved the paper. MK and $A B$ and $A M$ and FA and $A A$ and $S F$ and $M C$ and $S C$ and $L F$ and $M L$ and $J M$ and $B M$ and RM SO and $C O ̈$ and $B P$ and $I S$ and $W S$ and $A V$ and $X W$ and $S L$ and $C B$ and $S W$ and $J S$ and $M J$ and $Y N$ and ME JF and TZ gave guidance and improved the paper. All authors read and approved the final manuscript.

\section{Authors' information \\ N/A}

\section{Funding}

This study was endorsed by the ESICM. Free support for running the electronic database and was granted from the dep. of Epidemiology, University of Aarhus, Denmark. Financial support for creation of the e-CRF and maintenance of the database was possible from a grant (open project support) by Western Health region in Norway) 2018 who also funded the participating Norwegian ICUs. DRC Ile de France and URC Est helped conducting VIP2 in France. Open Access funding enabled and organized by Projekt DEAL.

\section{Availability of data and materials}

The anonymised data can be requested from the authors if required. The datasets analysed during the current study are not publicly available due to the different local institutional and/or licensing committees but are available from the corresponding author on reasonable request.

\section{Declarations}

\section{Ethics approval and consent to participate}

The primary competent ethics committee was the Ethics Committee of the University of Bergen, Norway. A study protocol was provided to participating centres. Every participating centre obtained ethics approval according to local legislation. A copy of the ethics approval was sent to the study coordinator before start of the study. Institutional research ethic board approval was obtained from each study site. This was a prerequisite for participation in the study. All methods were carried out in accordance with relevant guidelines and regulations. All experimental protocols were approved by the local institutional and/or licensing committees. Written informed consent was obtained of all included subjects, except for patients from VIP2 of sites where study inclusion was explicitly granted without written informed consent. The inclusion of deceased patients was strictly in accordance with the requirements of the local competent ethics committees. In most cases, the consent of the patient or the legal guardian was mandatory (see above). The studies conducted were observational studies. No examinations (e.g. blood sampling) or tissue sampling took place.

\section{Consent for publication}

The manuscript does not contain any individual person's data in any form.

\section{Competing interests}

The authors declare that they have no competing interests.

\section{Author details}

'Department of Cardiology, Pulmonary Diseases, and Vascular Medicine, Medical Faculty, Heinrich Heine University of Duesseldorf, Moorenstraße 5, 40225 Duesseldorf, Germany. ${ }^{2}$ Department of Anaesthesiology, Perioperative Medicine and Intensive Care Medicine, Paracelsus Medical University, Salzburg, Austria. ${ }^{3}$ Division of Cardiology, Department of Medicine, Karolinska Institutet, Karolinska University Hospital, Stockholm, Sweden. ${ }^{4}$ Cardiovascular Research Institute Düsseldorf (CARID), Duesseldorf, Germany. ${ }^{5}$ Service de Réanimation Médicale, Publique-Hôpital de Paris, Hôpital Saint-Antoine, 
F-75012 Paris, France. ${ }^{6}$ Department of Rehabilitation Hospital Ancelle di Cremona, Cremona, Italy. ${ }^{7}$ Geriatric Research Group, Brescia, Italy. ${ }^{8}$ Department Of Anaesthesia and Intensive Care, Ålesund Hospital, Ålesund, Norway. ${ }^{9} \mathrm{NTNU}$, Dep of Circulation and Medical Imaging, Trondheim, Norway. ${ }^{10}$ Department of Intensive Care Medicine, CIBERes Corporacion Sanitaria Universitaria Parc Tauli, Barcelona, Spain. ${ }^{11}$ Istituto di Ricerche Farmacologiche Mario Negri IRCCS, Ranica, BG, Italy. ${ }^{12}$ Department of Anaesthesia, IRCCS Instituto Clínico Humanitas, Humanitas University, Milan, Italy. ${ }^{13}$ Department of Anaesthesia and Intensive Care Medicine, Aarhus University Hospital, Aarhus, Denmark. ${ }^{14}$ Grande Ospedale Metropolitano Niguarda, Milan, Italy. ${ }^{15}$ Department of Cardiology, Paracelsus Medical University, Salzburg, Austria. ${ }^{16}$ Mater Misericordiae University Hospital, Dublin, Ireland. ${ }^{17}$ Unidade de Cuidados Intensivos Neurocríticos e Trauma, Faculdade de Ciências Médicas de Lisboa, Hospital de São José, Centro Hospitalar Universitário de Lisboa Central, Nova Médical School, Lisbon, Portugal. ${ }^{18}$ Department of Intensive Care, 1K12IC Ghent University Hospital, Ghent, Belgium. ${ }^{19}$ Karolinska University Hospital, Solna, Sweden. ${ }^{20}$ Department of Acute Medicine, Geneva University Hospitals, Geneva, Switzerland. ${ }^{21}$ Department of Intensive Care Medicine, University Medical Center, University Utrecht, Utrecht, The Netherlands. ${ }^{22}$ Intensive Care and Perioperative Medicine Division, Jagiellonian University Medical College, Kraków, Poland. ${ }^{23}$ Kardinal Schwarzenberg Hospital, Schwarzach, Austria. ${ }^{24} \mathrm{St}$ George's University Hospital, London, UK. ${ }^{25}$ Research Lead Critical Care Directorate St George's Hospital, London, UK. ${ }^{26}$ NAHP Committee ESICM, Intensive Care Unit, Royal Devon \& Exeter NHS Foundation Trust, Exeter, UK. ${ }^{27}$ Linkoping University Hospital, Linkoping, Sweden. ${ }^{28}$ Department of Intensive Care Medicine, Inselspital, Universitätsspital, University of Bern, Bern, Switzerland. ${ }^{29}$ Division of Intensive Care and Emergency Medicine, Department of Internal Medicine, Medical University Innsbruck, Innsbruck, Austria. ${ }^{30}$ European Wellness International, ICU, Luhansk, Ukraine. ${ }^{31}$ Alkhums Hospital, ICU, Tripoli, Libya. ${ }^{32}$ Department of Intensive Care, Aarhus University Hospital, Aarhus, Denmark. ${ }^{33}$ Intensive Care Unit General Hospital of Larissa, Larissa, Greece. ${ }^{34}$ Sorbonne Universités, UPMC Univ Paris 06, UMR_S 1136, Institut Pierre Louis d'Epidémiologie et de Santé Publique, F-75013 Paris, France. ${ }^{35}$ INSERM, UMR_S 1136, Institut Pierre Louis d'Epidémiologie et de Santé Publique, F-75013 Paris, France. ${ }^{36}$ Department of Clinical Medicine, University of Bergen, Bergen, Norway. ${ }^{37}$ Department of Anaestesia and Intensive Care, Haukeland University Hospital, Bergen, Norway.

Received: 13 January 2021 Accepted: 17 September 2021

Published online: 19 October 2021

\section{References}

1. Kontis V, Bennett JE, Mathers CD, Li G, Foreman K, Ezzati M. Future life expectancy in 35 industrialised countries: projections with a Bayesian model ensemble. Lancet. 2017;389(10076):1323-35. https://doi.org/10.1016/S01406736(16)32381-9.

2. Arenal JJ, de Teresa G, Tinoco C, Toledano M, Said A. Abdominal surgery in nonagenarians: short-term results. Surg Today. 2007;37(12):1064-7. https:// doi.org/10.1007/s00595-007-3537-1.

3. Boumendil A, Somme D, Garrouste-Orgeas M, Guidet B. Should elderly patients be admitted to the intensive care unit? Intensive Care Med. 2007; 33(7):1252. https://doi.org/10.1007/s00134-007-0621-3.

4. Guidet B, de Lange DW, Flaatten H. Should this elderly patient be admitted to the ICU? Intensive Care Med. 2018;44(11):1926-8.

5. Flaatten H, Garrouste-Orgeas M. The very old ICU patient: a never-ending story. Intensive Care Med. 2015;41(11):1996-8. https://doi.org/10.1007/ s00134-015-4052-2.

6. Guidet B, Leblanc G, Simon T, Woimant M, Quenot JP, Ganansia O, et al. Effect of systematic intensive care unit triage on long-term mortality among critically ill elderly patients in France: a randomized clinical trial. JAMA. 2017;318(15):1450-9. https://doi.org/10.1001/jama.201 7.13889.

7. Chin-Yee N, D'Egidio G, Thavorn K, Heyland D, Kyeremanteng K. Cost analysis of the very elderly admitted to intensive care units. Crit Care. 2017; 21(1):109. https://doi.org/10.1186/s13054-017-1689-y.

8. Ihra GC, Lehberger J, Hochrieser H, Bauer P, Schmutz R, Metnitz B, et al. Development of demographics and outcome of very old critically ill patients admitted to intensive care units. Intensive Care Med. 2012;38(4): 620-6. https://doi.org/10.1007/s00134-012-2474-7.
9. Fuchs L, Chronaki CE, Park S, Novack V, Baumfeld Y, Scott D, et al. ICU admission characteristics and mortality rates among elderly and very elderly patients. Intensive Care Med. 2012;38(10):1654-61. https://doi.org/10.1007/ s00134-012-2629-6.

10. Somme D, Maillet JM, Gisselbrecht M, Novara A, Ract C, Fagon JY. Critically ill old and the oldest-old patients in intensive care: short- and long-term outcomes. Intensive Care Med. 2003;29(12):2137-43. https://doi.org/10.1007/ s00134-003-1929-2.

11. Roedl K, Jarczak D, Becker S, Fuhrmann V, Kluge S, Muller J. Long-term neurological outcomes in patients aged over 90 years who are admitted to the intensive care unit following cardiac arrest. Resuscitation. 2018;132:6-12. https://doi.org/10.1016/j.resuscitation.2018.08.020.

12. Le Gall JR, Neumann A, Hemery F, Bleriot JP, Fulgencio JP, Garriques B, et al. Mortality prediction using SAPS II: an update for French intensive care units. Crit Care. 2005;9(6):R645-52. https://doi.org/10.1186/cc3821.

13. Guidet B, de Lange DW, Boumendil A, Leaver $S$, Watson X, Boulanger $C$, et al. The contribution of frailty, cognition, activity of daily life and comorbidities on outcome in acutely admitted patients over 80 years in European ICUs: the VIP2 study. Intensive Care Med. 2019;132:6-12.

14. Flaatten $\mathrm{H}$, De Lange DW, Morandi A, Andersen FH, Artigas A, Bertolini G, et al. The impact of frailty on ICU and 30-day mortality and the level of care in very elderly patients ( $>/=80$ years). Intensive Care Med. 2017;43(12): 1820-8. https://doi.org/10.1007/s00134-017-4940-8.

15. D'Agostino RB Jr. Propensity scores in cardiovascular research. Circulation. 2007;115(17):2340-3. https://doi.org/10.1161/CIRCULATIONA HA.105.594952.

16. Guidet B, Flaatten $\mathrm{H}$, Boumendil A, Morandi A, Andersen FH, Artigas A, et al. Withholding or withdrawing of life-sustaining therapy in older adults $(>/=$ 80 years) admitted to the intensive care unit. Intensive Care Med. 2018; 44(7):1027-38. https://doi.org/10.1007/s00134-018-5196-7.

17. Muessig JM, Nia AM, Masyuk M, Lauten A, Sacher AL, Brenner T, et al. Clinical frailty scale (CFS) reliably stratifies octogenarians in German ICUs: a multicentre prospective cohort study. BMC Geriatr. 2018;18(1):162. https:// doi.org/10.1186/s12877-018-0847-7.

18. Jung C, Wernly B, Muessig JM, Kelm M, Boumendil A, Morandi A, et al. A comparison of very old patients admitted to intensive care unit after acute versus elective surgery or intervention. J Crit Care. 2019;52:141-8. https:// doi.org/10.1016/j.jcrc.2019.04.020

19. Rockwood K, Song X, MacKnight C, Bergman H, Hogan DB, McDowell I, et al. A global clinical measure of fitness and frailty in elderly people. CMAJ. 2005;173(5):489-95. https://doi.org/10.1503/cmaj.050051.

20. Jorm AF, Jacomb PA. The informant questionnaire on cognitive decline in the elderly (IQCODE): socio-demographic correlates, reliability, validity and some norms. Psychol Med. 1989;19(4):1015-22. https://doi.org/10.1017/ S0033291700005742.

21. Katz S. Assessing self-maintenance: activities of daily living, mobility, and instrumental activities of daily living. J Am Geriatr Soc. 1983;31(12):721-7. https://doi.org/10.1111/j.1532-5415.1983.tb03391.x.

22. Evans DC, Cook CH, Christy JM, Murphy CV, Gerlach AT, Eiferman D, et al. Comorbidity-polypharmacy scoring facilitates outcome prediction in older trauma patients. J Am Geriatr Soc. 2012;60(8):1465-70. https://doi.org/1 0.1111/j.1532-5415.2012.04075.x.

23. Jones A, Toft-Petersen AP, Shankar-Hari M, Harrison DA, Rowan KM. Demographic Shifts, Case Mix, Activity, and Outcome for Elderly Patients Admitted to Adult General ICUs in the United Kingdom, Wales, and Northern Ireland. Crit Care Med. 2020;48(4):466-74.

24. Kim C, Becker L, Eisenberg MS. Out-of-hospital cardiac arrest in octogenarians and nonagenarians. Arch Intern Med. 2000;160(22):3439-43. https://doi.org/10.1001/archinte.160.22.3439.

25. Druwe P, Benoit DD, Monsieurs KG, Gagg J, Nakahara S, Alpert EA, et al. Cardiopulmonary resuscitation in adults over 80 : outcome and the perception of appropriateness by clinicians. J Am Geriatr Soc. 2020;68(1):3945. https://doi.org/10.1111/jgs.16270.

26. Becker S, Muller J, de Heer G, Braune S, Fuhrmann V, Kluge S. Clinical characteristics and outcome of very elderly patients $>/=90$ years in intensive care: a retrospective observational study. Ann Intensive Care. 2015; 5(1):53. https://doi.org/10.1186/s13613-015-0097-1.

27. Demoule A, Cracco C, Lefort Y, Ray P, Derenne JP, Similowski T. Patients aged 90 years or older in the intensive care unit. J Gerontol A Biol Sci Med Sci. 2005;60(1):129-32. https://doi.org/10.1093/gerona/60.1.129. 
28. Boumendil A, Aegerter P, Guidet B, Network CU-R. Treatment intensity and outcome of patients aged 80 and older in intensive care units: a

multicenter matched-cohort study. J Am Geriatr Soc. 2005;53(1):88-93. https://doi.org/10.1111/j.1532-5415.2005.53016.x.

\section{Publisher's Note}

Springer Nature remains neutral with regard to jurisdictional claims in published maps and institutional affiliations.

Ready to submit your research? Choose BMC and benefit from:

- fast, convenient online submission

- thorough peer review by experienced researchers in your field

- rapid publication on acceptance

- support for research data, including large and complex data types

- gold Open Access which fosters wider collaboration and increased citations

- maximum visibility for your research: over $100 \mathrm{M}$ website views per year

At $\mathrm{BMC}$, research is always in progress.

Learn more biomedcentral.com/submissions 